\title{
Wave-by-wave control in irregular waves for a wave energy converter with approximate parameters
}

\author{
Umesh A. Korde ${ }^{1}$ • Rush D. Robinett III ${ }^{2}$ - David G. Wilson ${ }^{3}$
}

Received: 30 March 2016 / Accepted: 3 October 2016 / Published online: 20 October 2016

(C) Springer International Publishing Switzerland 2016

\begin{abstract}
This paper investigates wave-by-wave control of a single-mode wave energy converter driven to operate such that the oscillation velocity closely matches the hydrodynamically optimum velocity for best power absorption. Such control typically requires prediction of the incident wave profile, which, for realistic wave spectra may be obtained using up-wave measurements over a duration and at a distance based on a deterministic propagation model and the device dynamics. This work investigates how such control may be attempted when the device inertia, viscous damping, hydrostatic stiffness, frequency-dependent hydrodynamic coefficients, and exciting force are quantified approximately. In particular, this paper studies an implementation of adaptive trajectory-tracking control using on-line estimation of the mechanical and hydrodynamic parameters (i.e. inertia, viscous damping, hydrostatic stiffness, frequency-dependent added mass, frequency-dependent radiation damping, and the exciting force), where a hydrodynamically optimum velocity variation based on approximate parameter estimates provides the reference trajectory. In this study, the rest mass, infinitefrequency added mass, hydrostatic stiffness, a linearized
\end{abstract}

$\triangle$ Umesh A. Korde

Umesh.Korde@sdsmt.edu

Rush D. Robinett III

rdrobine@mtu.ed

David G. Wilson

dwilso@sandia.gov

1 Department of Mechanical Engineering, South Dakota School of Mines and Technology, Rapid City, SD 57701, USA

2 Department Mechanical Engineering-Engineering Mechanics, Michigan Technological University, Houghton, MI 49931, USA

3 Department of Electrical Science and Experiments, Sandia National Laboratories, Albuquerque, NM 87185, USA viscous damping coefficient, and two parameters representing the uncertainties in the radiation impulse-response function and the exciting force impulse-response function are estimated on line. The present method relies on feedback and feedforward forces derived using a Lyapunov function comprised of a system Hamiltonian that combines the mechanical and information exergy functions. Energy capture results under oscillation constraints show that, while the present implementation leaves significant room for improvement relative to near-optimal wave-by-wave control with exact parameters, considerable improvement is still observed relative to resistive control with exact parameters.

Keywords Wave energy conversion - Wave-by-wave control - Irregular waves · Parameter uncertainty · Adaptive control · Exergy

\section{List of symbols}

$[\Gamma]$ Diagonal matrix of adaptation gains

$\Delta t \quad$ Update rate for parameter estimates

$\eta\left(x_{B} ; t\right)$ Incident wave surface elevation at buoy centroid

$\begin{array}{ll} & x_{B} \\ \lambda_{w} & \text { Wave length }\end{array}$

$\rho \quad$ Density of water

$\{\phi\} \quad$ Parameter estimate error vector

$A(\omega) \quad$ Wave amplitude

$b(\omega) \quad$ Frequency dependent radiation damping in heave

$b_{h}(\omega) \quad$ Approximate frequency dependent radiation damping

$c_{d} \quad$ Linearized viscous damping coefficient in heave

$D_{r} \quad$ Buoy draft

$F_{f} \quad$ Exciting force on buoy in heave

$F_{L} \quad$ Total actuator force applied on buoy 
$F_{r} \quad$ Feedforward force

$F_{f b} \quad$ Feedback force

$F_{f k} \quad$ Froude-Krylov approximation to the exciting force in heave

$g \quad$ Acceleration of gravity

$h_{a}(t) \quad$ Odd-function part of $h_{r}(t)$

$H_{f}(i \omega)$ Frequency response function for exciting force $F_{f}$ in heave

$h_{f}(t) \quad$ Impulse-response function for the exciting force in heave

$h_{l}(t) \quad$ Impulse-response function describing the linear uni-directional wave propagation process in deep water

$h_{r}(t) \quad$ Causal radiation impulse-response function

$h_{b}(t) \quad$ Even function part of $h_{r}(t)$

$H_{S} \quad$ Significant wave height

$h_{f h}(t)$ Approximate expression for the exciting force impulse-response function

$h_{r h}(t) \quad$ Approximate expression for the radiation impulse response function

$k \quad$ Hydrostatic stiffness coefficient for the buoy heave

$K_{a} \quad$ Feedback gain attached to acceleration error

$K_{x} \quad$ Feedback gain attached to position error

$m \quad$ In-air mass of the buoy

$P_{w} \quad$ Incident wave power per unit crest length

$R_{S} \quad$ Buoy radius

$T_{e} \quad$ Energy period

$T_{w} \quad$ Wave period

$v \quad$ Heave velocity

$x \quad$ Heave displacement

$\mathcal{H} \quad$ System Hamiltonian, expressing the total exergy

$\mathcal{H}_{i} \quad$ Information exergy

$\mathcal{H}_{m} \quad$ Mechanical exergy

$\hat{c}_{d} \quad$ Approximate estimate for $c_{d}$

$\hat{F} \quad$ Single multiplicative parameter assumed to relate $h_{f h}$ and $h_{f}$

$\hat{k} \quad$ Approximate estimate for $k$

$\hat{M} \quad$ Approximate estimate for $m+\bar{a}(\infty)$

$\hat{R} \quad$ Single multiplicative parameter assumed to relate $h_{r h}$ and $h_{r}$

$\bar{a}(\infty) \quad$ Infinite-frequency added mass for the buoy in heave

$\bar{a}(\omega) \quad$ Frequency-dependent added mass inclusive of the infinite-frequency added mass $\bar{a}(\infty)$

\section{Introduction}

Wave energy converters utilizing relative oscillation between a wave-activated body and a reference have received considerable attention in the literature for a long time now (see, for instance, Falcão 2010). Floating bodies in heave, pitch, or roll have distinct natural frequencies due to their inertia and hydrostatic restoring force/moments. Hence, they tend to perform efficiently when the peak-frequencies in the incoming spectra are close to the body natural frequencies. For single-mode devices without viscous damping or oscillation constraints, maximum conversion requires the body to resonate, and in addition, the energy absorption rate must match the frequency-dependent energy radiation rate from body oscillation. Consequently, without hydrodynamic control, converters frequently may get bulky and the annually averaged energy conversion rates may not be high enough for cost effectiveness.

Early implementations of control in the 1970s involved adjustable resonant tuning via reactive (negative spring or positive inertia) loads (Salter 1978), and latching-type switching control. Latching control used braking forces to lock and release buoy oscillation so as to force the velocity (when unlocked) to be synchronous with exciting force (Budal and Falnes 1980). Complex conjugate control generalizes the control of Salter (1978), and consists of using the power take-off (together with another actuator if required) to apply a resistive load that matches the radiation damping for the floating body, and in addition, applying a reactive load that cancels the reactive part of the converter impedance (i.e. due to stiffness and inertia of the floating body). While such impedance matching implies maximum power absorption in regular waves, extension of such control to irregular waves presents fundamental challenges (Naito and Nakamura 1985; Falnes 1995). Non-real time 'peak frequency tuning' type approaches have been attempted with success in practice (Hansen and Kramer 2011), where simply matching impedances at the peak frequency of incoming spectra at regular intervals could lead to a three-fold improvement in annual energy production. Such reactive control requires an actuation system capable of exchanging reactive power with the floating body, and of drawing on stored energy.

The challenges of implementing the frequency-domain notion of complex-conjugate control on a wave-by-wave basis for wave energy devices arise in part from the nature of the radiation force produced on the body by the waves generated by the body. This is because the radiation force at any given instant includes not only the contribution of waves created at that instant but also that of waves created at prior instants. It is only the present and past oscillations that determine the radiation force, however, so the impulse-response function $h_{r}$ describing it is causal. Therefore, its Fourier transform is analytic in the upper half of the complex half plane, which implies that the real and imaginary parts of its Fourier transform are constrained by the Kramers-Kronig relations (Wehausen 1992). Complex conjugate control requires that the real part be matched and the imaginary part be cancelled using actuator loads. Thus, the odd function part $h_{a}$ and the negative of the even function 
part $h_{b}$ of $h_{r}=h_{a}+h_{b}$ need to be synthesized separately in order to generate the real-time control force to be applied (on the body from the reference). Because both $h_{a}$ and $h_{b}$ are non-causal, velocity information from the future is required for complete synthesis of the control force. This situation is discussed in Naito and Nakamura (1985), and Falnes (1995). In addition, because of the continuum nature of incident wave action and the body's geometric size, the body begins to 'feel' an exciting force somewhat before an incident wave arrives at its centroid. Consequently, the impulse-response function $h_{f}$ describing the exciting force (relative to the incident wave profile at the body centroid) in the time domain is non-causal (Falnes 1995). Application of 'complex conjugate control' in real time therefore requires knowledge of the incident wave profile (and device oscillation), typically, 2030 seconds in advance. Approximate approaches based on time-series analysis of past oscillations have been attempted (e.g. see Korde 1999, and more recent works such as, Fusco and Ringwood 2010). A number of other approximate control approaches have been developed in recent years (see Hals et al. 2011) for a comparative assessment). Direct use of incident wave profile measurement some distance up-wave for generating the control forces at the current instant has also been reported (Korde 2014). More recently, near-optimal wave-by-wave control was investigated (Korde 2015), for which a deterministic propagation model was used to predict the wave surface elevation at the device up to the required duration into the future. In that work, a wave measurement made over a prescribed time duration and at a prescribed distance in the up-wave direction was used in a convolution integral whose kernel was the impulse-response function representing the linear wave propagation model. The up-wave distance and the time duration of measurement used in the convolution were determined from the group-velocity range associated with commonly encountered practical sea-states and the device dynamics.

Considerable room for improvement exists in at least two areas: (1) increasing accuracy with alternative approaches to utilize up-wave surface elevation or other measurements for evaluating control forces, and/or (2) finding ways to deal with uncertainties in the physical and hydrodynamic parameters describing the converter dynamics. The work reported in this paper is relevant to the second area, and is relevant for the following reasons: Although most converter models assume perfect knowledge of the in-air mass and moments of inertia, viscous damping, and hydrostatic stiffness associated with the floating body, these parameters may only be approximately known in practice. Actuator and load inertias, and actuator dissipation effects are not considered in many device models. Load inertias may change during device operation, and actuator dissipation effects may only approximately be known. For floating bodies where water-plane areas change with displacement, the use of linearized stiffness coefficients is at best approximate. It is also common to use linearized viscous damping coefficients to approximate viscous friction. Such approximations have limited applicability when oscillation (displacement and velocity) amplitudes are large. Similarly, linearized actuator models may also lose applicability in large oscillations. Recall that oscillation amplitudes become large when impedance matching conditions are approached. Further, the numerically evaluated hydrodynamic quantities such as exciting force impulse-response function, the radiation force impulse-response functions, and the infinite-frequency added masses/inertias are also only good approximations valid for small oscillations. Nevertheless, linear models and linear design techniques take less effort and provide significant insight, to a level of generality not possible with nonlinear models. For this reason, it may be worthwhile to use a linear modeling and design framework wherein the parameters are thought to be imprecisely known, but wherein parameter estimates are updated on a slower time-scale. It is relevant here to point out the use of multiple-scale methods where higher order effects may be thought to be varying at slower time scales (e.g see Mei 1992).

Recent approaches seeking control in the presence of uncertainties in the dynamic model include the robust controller approach investigated in Fusco and Ringwood (2014). In another recent work, wave-by-wave control using a linear model was attempted in the presence of uncertainties in the rest mass, stiffness, and linearized viscous damping, but it was assumed that the frequency variations of the hydrodynamic coefficients and the exciting force would be perfectly known (Korde et al. 2015). The present paper also uses a linear model with uncertainly known coefficients for rest mass, hydrostatic stiffness, and viscous friction damping but in addition also allows for the use of approximate estimates for the frequency-dependent hydrodynamic coefficients and the exciting force. Uncertainties in wave prediction are not considered in this paper. In order to allow a comparison between the present approach and wave-by-wave control with perfectly known, unchanging device dynamics, the exact rest mass, hydrostatic stiffness, and viscous damping coefficient were assumed to be available. Approximately known starting estimates for these parameters were assumed to be within $\pm 20 \%$. As the 'exact' hydrodynamic coefficients and exciting force were numerically determined, the hydrodynamic coefficients and exciting force as determined based on a Froude-Frylov approximation (i.e. diffraction effects ignored) were here used as starting estimates. It should also be pointed out that, if the proposed approach can be implemented successfully, for devices with complex geometries, one could conceivably use the hydrodynamic coefficients and exciting forces for a simpler representative geometry in control design if exact numerical values are not immediately available. Note, finally, that the exact physical 
parameters (i.e. mass, stiffness, etc.) and hydrodynamic parameters (hydrodynamic coefficients, and exciting force) will generally not be available in a practical implementation. The 'exact' values are not needed for the present method, but are here assumed to be available in order to allow comparison of results.

As mentioned, this paper investigates a control strategy that approximates wave-by-wave impedance matching control in the presence of parameter uncertainty. Wave-profile prediction up to some time into the future is required, and the control force includes both feedforward and feedback forces. Also as mentioned, wave prediction based on the approach of Korde (2015) was assumed to be available. Even though the approach of Korde (2015) is limited to long-crested waves, errors arising from this restriction and from the linear deterministic model are considered outside the scope of the present study (see, however, Korde et al. 2016). Note, however, that primarily uni-directional, long-crested waves may be observed over entire days at some sites (e.g. NDBC 2016). The overall procedure could be extended to incorporate inaccuracies in wave prediction, although such an extension is not considered here. The goal of the present overall formulation is to enable trajectory tracking and on-line parameter estimation, while using the improving parameter estimates in determining the instantaneous control force. The trajectory to be tracked is computed using an impulse-response function based on the available approximate estimates for the exciting force, radiation damping, and viscous damping ('estimated reference trajectory').

This paper addresses uncertainties in (i) the rest mass, (ii) linearized viscous damping coefficient, (iii) linearized hydrostatic stiffness, (iv) frequency-dependent added mass, (v) frequency-dependent radiation damping, and (vi) the frequency-dependent exciting force. Note that the present study uses impulse-response functions based on the uncertainly known frequency-dependent parameters in (iv)-(vi). The feedforward force at each instant is based on the current estimates for the in-air mass, stiffness coefficient, linearized viscous damping coefficient, infinite-frequency added mass, frequency-variable added mass (defined here as in Korde 2015), the impulse-response function $h_{a}$, and the impulseresponse function $h_{b}$ (where $h_{a}(t)+h_{b}(t)=h_{r}(t)$, the radiation impulse-response function. Here $h_{a}$ is an odd function of $t$, while $h_{b}$ is an even function of $t . h_{r}(t)$ is a causal function of $t$, i.e. $h_{r}(t)=0, t<0$.), and the estimated reference velocity (the evaluation of the feedforward force therefore also requires wave-profile prediction). The feedback force is based on the difference between the estimated reference trajectory and the actual trajectory (position, velocity, and acceleration signals are used). Feedback gains used in this procedure are held constant over all simulation cases studied here. Parameter estimates are specific to each run, and are updated at periodic intervals according to an 'adaptation law' that is derived to minimize error. The reference trajectory is also updated as parameter estimates improve. Sections 2, 3, 4 describe the dynamic model and the overall waveby-wave control technique which combines the two goals of trajectory tracking and parameter estimation. The overall combined trajectory-tracking and parameter estimation approach has been tested in a number of situations outside of wave energy conversion, namely, in flexible-robot control, temperature control in buildings, ship-board power management, etc. (Robinett et al. 2002; Robinett and Wilson 2011; Slotine and Li 1991; Bryson and Ho 1975, etc).

Section 2 following this introduction summarizes waveby-wave near-optimal control based on wave prediction when exact information on all parameters is available. Section 3 discusses the manner in which parameter approximations are represented in this paper. Section 4 describes the overall adaptive estimation and control formulation. Calculations and simulations carried out here are described in Sect. 5, while the principal results of this work are discussed in Sect. 6. The paper concludes with a review of the main conclusions in Sect. 7.

\section{Wave-by-wave control with exact parameters}

To allow focus on the investigation of the overall modeling and control strategy, a cylindrical buoy in predominant heave oscillation is considered. Oscillations relative to a deeply submerged reaction mass (assumed stationary) are utilized for power conversion using a linear actuator, which may be either hydraulic (e.g. a double-acting hydraulic cylinder) or electric (a permanent magnet linear generator/motor). As required, additional actuators in parallel may be used to share in the application of the overall control force. Stored energy enough to support the required reactive power exchange in the form of hydraulic accumulators or batteries is assumed to be available, and further, the actuators are assumed to be linear and ideal in the implementation with exact parameters. Summarized here is an overview of the approach followed in Korde (2015), as applied to a single-body oscillator. Figure 1 shows a schematic view of the device geometry.

In Eq. (1) below, $m$ denotes the in-air mass of the buoy, $R_{S}$ the buoy radius, $D_{r}=R_{S} / 2$ the buoy draft, $\bar{a}(\infty)$ the infinitefrequency added mass in heave, $k=\rho g \pi R_{s}^{2}$ the hydrostatic stiffness coefficient for the buoy heave, $c_{d}$ the linearized viscous damping coefficient in heave, $v(t)$ the heave velocity at time $t$, and $F_{f}(t)$ the exciting force in heave. The heave oscillation of the buoy can be described using the relation,

$$
\begin{aligned}
& {[m+\bar{a}(\infty)] \dot{v}(t)+\int_{0}^{\infty} h_{r}(\tau) v(t-\tau) \mathrm{d} \tau+c_{d} v(t)} \\
& \quad+k \int_{-\infty}^{t} v(\tau) \mathrm{d} \tau=F_{f}(t)+F_{L}(t)
\end{aligned}
$$




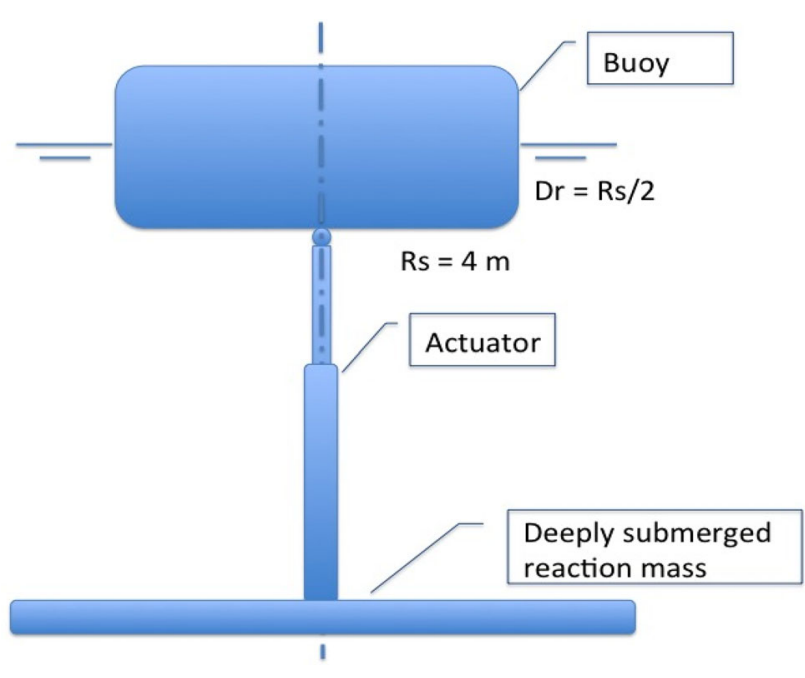

Fig. 1 The cylindrical buoy device studied in this work. The buoy is assumed to be in predominantly heave oscillation relative to the sea floor or a deeply submerged reaction mass, and a linear actuator (hydraulic cylinder or permanent magnet linear generator/motor) utilizes the buoy heave oscillation for energy conversion

$h_{r}(t)=\frac{2}{\pi} \int_{0}^{\infty} b(\omega) \cos \omega t \mathrm{~d} \omega=-\frac{2}{\pi} \int_{0}^{\infty} \omega a(\omega) \sin \omega t \mathrm{~d} \omega$

Equation (1) is an integro-differential equation (often called the 'Cummins' equation') (Cummins 1962). $b(\omega)$ is the frequency-dependent radiation damping in heave and $a(\omega)$ is defined as

$a(\omega)=\bar{a}(\omega)-\bar{a}(\infty)$

$\bar{a}(\omega)$, the frequency-dependent added mass in heave behaves as,

$\lim _{\omega \rightarrow \infty} \bar{a}(\omega) \rightarrow \bar{a}(\infty)$

$a(\omega)$ is thus the Fourier-transformable, frequency-variable part of $\bar{a}(\omega)$. Because $h_{r}(t)$ is real-valued and causal, $\omega a(\omega)$ and $b(\omega)$ are, respectively, odd and even functions of frequency, and satisfy the Kramers-Kronig relations. Their full inverse Fourier transforms can be defined as,

$h_{a}(t)=\frac{1}{2 \pi} \int_{-\infty}^{\infty} \omega a(\omega) \mathrm{e}^{i \omega t} \mathrm{~d} \omega$,

$h_{b}(t)=\frac{1}{2 \pi} \int_{-\infty}^{\infty} b(\omega) \mathrm{e}^{i \omega t} \mathrm{~d} \omega$

Note that $h_{a}(t)$ is an odd function of $t$ and $h_{b}(t)$ is an even function of $t$. Thus, $h_{r}(t)=h_{a}(t)+h_{b}((t)$ in the time domain, and hence, both $h_{a}$ and $h_{b}$ are non-causal. Therefore, their use in generating control forces on a wave-by-wave basis requires prediction of velocity $v$.

The exciting force $F_{f}(t)$, as commonly expressed in terms of the surface elevation at body centroid is,

$F_{f}(t)=\int_{-\infty}^{\infty} h_{f}(\tau) \eta\left(x_{B} ; t-\tau\right) \mathrm{d} \tau$

where $\tau$ is the dummy time variable over which the integration is performed, $\eta\left(x_{B} ; t\right)$ is the wave surface elevation at buoy centroid $x_{B}$, and $h_{f}(t)$ is the impulse-response function defining the exciting force in heave. Because wave action takes place over a continuum, and because it produces a pressure over the buoy surface before the incident wave reaches the centroid, $h_{f}(t)$ is also non-causal, and can be expressed as,

$h_{f}(t)=\frac{1}{2 \pi} \int_{-\infty}^{\infty} H_{f}(i \omega) \mathrm{e}^{i \omega t} \mathrm{~d} \omega$

Here $H_{f}(i \omega)$ is the frequency response function describing the exciting force frequency dependence for unit incident wave amplitude, given by $F_{f}(i \omega) / A$.

Note that the memory effect in $h_{r}$ can be approximated as a finite time interval on the order of $t_{r} \sim 10-20 \mathrm{~s}$. Both $h_{a}$ and $h_{b}$ therefore approximately only have support in the $\pm t_{r}$ range. Similarly, the non-causal part of $h_{f}$ also extends a finite duration $t_{f} \sim 10-20 \mathrm{~s}$. The integrals in Eqs. (5) and (7) can therefore be evaluated using finite limits in simulations. The approximate finiteness of $t_{r}$ and $t_{f}$ also results in a finite prediction-time for the wave surface elevation $\eta$ at $x_{B}$. However, as noted in Korde (2015), it is partly for these approximations that practical implementations of the wave-by-wave impedance matching control are at best nearoptimum.

For the buoy heave velocity to be at the hydrodynamic optimum (maximizing power transfer from the incident wave to the buoy), such that $v(t)=v_{o}(t)$ (Falnes 1995),

$2 c_{d} v_{o}(t)+2 \int_{-\infty}^{\infty} h_{b}(\tau) v_{o}(t-\tau) \mathrm{d} \tau=F_{f}(t)$

This condition can be achieved if the control force applied on the buoy is of the form (Korde 2015),

$$
\begin{aligned}
F_{L}(t)= & {[m+\bar{a}(\infty)] \dot{v}_{o}(t)+k \int_{-\infty}^{t} v_{o}(\tau) \mathrm{d} \tau } \\
& +\int_{-\infty}^{\infty} h_{a}(\tau) v_{o}(t-\tau) \mathrm{d} \tau-c_{d} v_{o}(t) \\
& -\int_{-\infty}^{\infty} h_{b}(\tau) v_{o}(t-\tau) \mathrm{d} \tau
\end{aligned}
$$


$F_{L}(t)$ can be seen to be a feedforward force, based on the desired velocity optimum $v_{o}(t)$. In practice, due to measurement errors and disturbances, a feedback controller will be required so that correct tracking is achieved, although the approach reviewed here is open loop, and measurement errors and disturbances were not accounted for. The hydrodynamic optimum $v_{o}(t)$ can be evaluated using,

$v_{o}(t)=\int_{-\infty}^{\infty} h_{o}(\tau) \eta\left(x_{B} ; t-\tau\right) \mathrm{d} \tau$

where $h_{o}(t)$ is a non-causal impulse-response function given by,

$h_{o}(t)=\frac{1}{2 \pi} \int_{-\infty}^{\infty} \frac{H_{f}(i \omega)}{2\left[c_{d}+b(\omega)\right]} \mathrm{e}^{i \omega t} \mathrm{~d} \omega$

In the presence of an oscillation constraint that requires the maximum excursion to be less than a specified limit, (e.g. the draft or the freeboard to avoid full emergence or full submergence), the frequency-domain approach of Evans (1981) may be used as implemented in Korde (2015). Briefly, this corresponds to redefining the non-causal impulse-response function $h_{o}$ in Eq. (11) as

$h_{o c}(t)=\frac{1}{2 \pi} \int_{-\infty}^{\infty} \frac{H_{f}(i \omega)}{2\left[c_{d}+\Lambda(\omega)+b(\omega)\right]} \mathrm{e}^{i \omega t} \mathrm{~d} \omega$

The constrained optimum velocity can then be expressed as,

$v_{o c}(t)=\int_{-\infty}^{\infty} h_{o c}(\tau) \eta\left(x_{B} ; t-\tau\right) \mathrm{d} \tau$

Note that this approach for specifying the oscillation constraint limits the significant wave heights up to which the constraint will be satisfied Korde (2015). $\Lambda(\omega)$ is a dampinglike parameter. The advantage with this approach is that the oscillation constraint can be applied without resorting to inequality relations or comparisons within or outside of the control formulation. The memory effect of $h_{o}(t)$ requires the surface elevation time history going back into the past, and its non-causality requires surface elevation prediction up to a duration into the future that equals the non-zero tail length of $h_{o}(t)$ into $t<0$ (note that $h_{o}(t) \rightarrow 0$ asymptotically beyond some $t<-t_{f}$, and $t_{f}$ is approximate). Because $h_{a}(t)$ and $h_{b}(t)$ in Eq. (9) are also noncausal and both approach zero asymptotically beyond $t<-t_{p}$ ( $t_{p}$ is again approximate), evaluation of $F_{L}(t)$ requires prediction of $\eta\left(x_{B} ; t\right)$ up to $t_{f}+t_{p}$ into the future. In long-crested waves such a prediction may be obtained using a wave-elevation measurement made over a duration $T$ and at an up-wave distance $d$ Korde (2015), determined according to the range of group velocities $v_{g m n} \leq v_{g} \leq v_{g m x}$ encountered in practical wave spectra. Thus, with $d=x_{B}-x_{A}$ where $x_{A}$ is the point of up-wave measurement, then $\eta\left(x_{B} ; t+t_{p}\right)$ can be predicted using

$\eta\left(x_{B} ; t+t_{p}\right)=\int_{0}^{T} h_{l}(\tau) \eta\left(x_{A} ; t-\tau\right) \mathrm{d} \tau$

$x_{B}-x_{A}=d=t_{P} v_{g m x}$

$T=\frac{\mathrm{d}}{v_{g m n}}-\frac{\mathrm{d}}{v_{g m x}} ; t>T$

where deep-water conditions are assumed. $T$ is the time window up to the current instant over which past wave profile measurements at $x_{A}$ are needed. The lower and upper integration limits in Eq. (10) can in realistic situations be replaced by $-t_{p}$ and the current time $t$, respectively.

If all physical parameters $\left(m, k, c_{d}\right)$ are assumed perfectly known and the exact hydrodynamic coefficients and exciting force in heave are also perfectly known, then within linear theory, the procedure above is expected to provide nearoptimal wave-by-wave control through impedance matching in uni-directional, small-amplitude waves (near-optimal in view of the approximate determination of $t_{f}, t_{p}$, the small non-causality of the propagation impulse-response $h_{l}$ being ignored, and the group-velocity range being defined based on the frequency-range commonly observed with most wave spectra). Note, however, that certain operating sites may receive largely uni-directional waves over long periods, and similarly, a study of available historical spectral data at that site may also help to refine the spectral frequency and groupvelocity ranges [e.g. see NDBC (2016)].

\section{Use of approximate parameters}

The approach reviewed in Sect. 2 assumed perfect parameter knowledge. As indicated earlier, it is perhaps more common in practice to have only approximate knowledge of device parameters. The question addressed in the present study is: how wave-by-wave impedance matching control could be approximated in the presence of approximate knowledge of the physical parameters $\left(m, k, c_{d}\right)$, the hydrodynamic coefficients $\bar{a}(\omega), b(\omega)$, and the exciting force $H_{f}(i \omega)$. It should be noted here that uncertainties in the hydrodynamic parameters will directly translate into uncertainties in the impulseresponse functions associated with them [see Eqs. (2) and (5)]. In addition, $\bar{a}(\omega)=\bar{a}(\infty)+a(\omega)$, so that the effective inertia of the device as stated in Eq. (1) is $m+\bar{a}(\infty)$. In the treatment of Sect. 4, only approximate parameter estimates are thought to be available for determining the control force. Table 1 summarizes the uncertainties accounted for in Sect. 4.

As mentioned in the introduction (Sect. 1), the estimates $\hat{M}$, etc. were updated iteratively. The estimates were thought 
Table 1 Parameter approximations accounted for in the present study

\begin{tabular}{llll}
\hline Parameter/function & Exact & Approximate & Estimates updated \\
\hline Effective inertia/mass & $m+\bar{a}(\infty)$ & $\hat{M}$ & $\hat{M}$ \\
Hydrostatic stiffness & $\mathrm{k}$ & $\hat{k}$ & $\hat{k}$ \\
Linearized viscous damping & $c_{d}$ & $\hat{c}_{d}$ & $\hat{c}_{d}$ \\
Radiation impulse-response function & $h_{r}(t)$ & $h_{r h}(t)$ & $h_{f h}(t)$ \\
Exciting force impulse-response function & $h_{f}(t)$ & $v_{r} \equiv \dot{x}_{r}[$ Eq. $(16)]$ & $\hat{R}, h_{r}=\hat{R} h_{r h}$ \\
Reference trajectory & $v_{o}(t)[$ Eq. (13)] & $v_{r}(t)$ \\
\hline
\end{tabular}

to be slowly varying, and were updated every $N_{u}$ iterations [ $\left(N_{u}=10\right)$, assuming that a 10 times slower rate of change]. In order to allow comparison with results with exact parameters, the exact parameters were assumed to be known here, even though they do not have to be known in practice for the proposed method to work. The starting estimates for $\hat{M}, \hat{c}_{d}$, and $\hat{k}$ were assumed to be $0.8-1.2$ times the exact values, though in practice, the choice would be based on the best available estimates or approximations. The numerically determined hydrodynamic coefficients and exciting force variations (and the impulse-response functions associated with them) were thought to be exact for the purpose of this work. The corresponding approximations as determined using the Froude-Krylov approximation (i.e. with the diffraction effects ignored and forces determined using incident potentials only) were used as initial estimates. For ease of implementation, a simplifying assumption was introduced, that the approximate variations were related to the exact variations via a single multiplicative constant as shown in column 4 of Table 1. The estimation and control approach used here is based on updating the multiplicative constants $\hat{R}$ and $\hat{F}$ in addition to $\hat{M}, \hat{k}$, and $\hat{c}_{d}$. Note that $\hat{R}=\hat{F}=1$ indicates exact match. The method used for determining the approximate impulse-response functions $h_{f h}$ and $h_{r} h$ is discussed in Appendix A.

The reference trajectory can be specified ahead of time if prediction of the incident wave elevation is available sufficiently far into the future. If perfect knowledge of the impulse-response functions, wave elevation, and velocity into the future were available, the reference trajectory would be the hydrodynamic velocity optimum defined by Eq. (8). $v_{o}(t)$ would then be found using Eq. (10). Knowing that $b(\omega)=\hat{R} b_{h}(\omega)$, if it is assumed that $\hat{c}_{d} \approx \hat{R} c_{d}$, an estimated reference trajectory $v_{r}(t)$ can be defined,

$v_{r}(i \omega)=\frac{H_{f h}(i \omega)}{2\left[\hat{c}_{d}+b_{h}(\omega)\right]} A(\omega)$

In the time domain, letting $h_{o h}(t)$ be the inverse Fourier transform of the term attached to $A(\omega)$ in Eq. (15), $v_{r}(t)=\int_{-\infty}^{\infty} h_{o h}(\tau) \eta\left(x_{B} ; t-\tau\right) \mathrm{d} \tau$

This is the desired rate of change of heave displacement $\dot{x}_{r}=$ $v_{r}$. The desired displacement $x_{r}$ at each instant is determined using numerical integration. Thus,

$x_{r}(t)=\left[v_{r}(t)+v_{r}(t-h)\right] h$

Here $h$ denotes the length of a single time step at which the actuator forces are updated. Recall that $v_{r}(t)$ is computed using Eq. (16). Note that evaluation of $v_{r}(t)$ requires prediction of $\eta\left(x_{B} ; t\right)$ up to $t_{t h}$ into the future, where $t_{t h}$ is such that $h_{o h}(t) \rightarrow 0, t<-t_{t h}$. In this paper, an accurate prediction is assumed to be available, though extensions to the formulation to relax these restrictions are expected to be reported in a future paper.

When perfect knowledge is available, $v_{r}=v_{o}$. In the procedure adopted in this work, as successive estimates for $\hat{F}$ and $\hat{R}$ become available, the reference trajectory is updated according to,

$\dot{x}_{r}(t+\Delta t) \equiv v_{r}(t+\Delta t)=\left(\frac{\hat{R}}{\hat{F}}\right) v_{r}(t) \equiv\left(\frac{\hat{R}}{\hat{F}}\right) \dot{x}_{r}(t)$

$\Delta t$ represents the update rate for the parameter estimates. Parameter estimates and trajectory updating may be carried out every $N_{u}$ iterations.

$\Delta t=N_{u} h$

In the simulations reported on in this paper, estimates are updated every 10 time steps (i.e. $N_{u}=10$ in this paper). With $h=5 \times 10^{-3} \mathrm{~s}$ in most simulations, $\Delta t=0.05 \mathrm{~s}$ in most simulations reported on in this paper.

It is recalled that under an oscillation constraint

$h_{o c}(t)=\frac{1}{2 \pi} \int_{-\infty}^{\infty} \frac{H_{f}(i \omega)}{2\left[c_{d}+\Lambda(\omega)+b(\omega)\right]} \mathrm{e}^{i \omega t} \mathrm{~d} \omega$

where $H_{f}(i \omega)=F_{f}(i \omega) / A(\omega)$, i.e. exciting force in heave per unit incident wave amplitude. The best knowledge avail- 
able here is thought to be in the form of $H_{f h}, b_{h}(\omega)$, and $\hat{c}_{d}$, however. Therefore, an approximate impulse-response function $h_{o c h}$ is here defined as,

$h_{o c h}(t)=\frac{1}{2} \int_{-\infty}^{\infty} \frac{H_{f h}(i \omega)}{2\left[\hat{c}_{d}+\Lambda(\omega)+b_{h}(\omega)\right]} \mathrm{e}^{i \omega t} \mathrm{~d} \omega$

Note that $H_{f}(i \omega)=\hat{F} H_{f h}(i \omega)$, where $H_{f h}(i \omega)=$ $F_{f k}(i \omega) / A(\omega)$. Note that $\Lambda(\omega)$ is typically specified by the designer and is therefore known a priori. In the present simulations, a more approximate implementation was used for convenience, where, a constant value $\Lambda=\alpha_{c}(\max [\Lambda(\omega)])$ was used for all $\omega$, with $\alpha_{c}=0.6$ in most simulations. Further, the constrained reference velocity $v_{r c}$ based on $h_{\text {ohc }}$ was also updated using Eq. (18).

\section{Adaptation and control formulation}

As mentioned previously, the goal of this work is investigate an approach that is designed to improve upon the available approximation to the mass, viscous damping coefficient, hydrostatic stiffness, the radiation impulse-response function, and the exciting force impulse-response function for heave oscillation of a cylindrical buoy, while attempting to provide a close approximation to a desired velocity variation. The control forces include both a feedforward component and a feedback component. The feedforward component at each time instant is derived using the available approximations to each of the parameters just mentioned and the reference trajectory defined by $v_{r}(t)$. The feedforward component $F_{r}(t)$ below therefore requires wave prediction. The actual velocity and actual displacement are defined as $v$ and $x$, respectively. These quantities may be measured on line using velocity and displacement sensors, and the feedback component $F_{f b}(t)$ below can be designed to minimize the differences $x-x_{r}$ and $v-v_{r}$. The feedback gains in this work are chosen to be constants independent of frequency or time, and hence evaluation of the feedback component does not require wave prediction.

As summarized in Table 1, in the formulation below, the estimate for the sum of the in-air mass and the infinitefrequency added mass $m+\bar{a}(\infty)$ is defined as $\hat{M}$. Similarly, the estimates for the stiffness constant $k$ and the linearized viscous damping constant are defined as $\hat{k}$ and $\hat{c}_{d}$, respectively. The estimates for the impulse-response functions representing the exciting force and the radiation force are found using hydrodynamic approximations (here, the smallbody Froude-Krylov force to represent the overall exciting force). The exact functions $h_{r}=h_{a}+h_{b}$, and $h_{f}$ are thought to be related to the approximations via single multiplicative estimates $\hat{R}$, and $\hat{F}$, respectively, [see Eqs. (53) and (60)]. As discussed in Sect. 3, the reference trajectory to be tracked is the hydrodynamic velocity optimum based on wave prediction. However, this trajectory here is based on the best available current parameter estimates $\hat{c}_{d}, \hat{R}$, and $\hat{F}$. The control formulation must therefore enable tracking of the best available estimate for the optimum velocity trajectory, while providing corrections to the available estimates for the parameters and the trajectory. The approach followed here is based on a Lyapunov function, for which the conditions for negative definiteness of the derivative are used to update the parameter estimates, subject to the dynamic model for the device response. The system Hamiltonian obtained by adding exergy functions associated with the device dynamics and estimate accuracy provides an effective candidate Lyapunov function for the present problem (see also Robinett et al. (2002), Robinett and Wilson (2011), etc. for applications in other fields). The term 'exergy' is here used in a more general sense than the traditional thermodynamic exergy. We note that the term exergy here extends the notion of thermodynamic exergy and represents the total energy available in the system that can be converted into useful work. For a mechanical system, this is the sum of the potential and kinetic energy associated with position and velocity, respectively. When parameters associated with the mechanical exergy are only approximately known, the total exergy can be expressed as a sum of the mechanical exergy and the information exergy associated with parameter-estimate uncertainties. The minimum-exergy solution is then expected to represent the true motion of the uncertainly-known system. More details can be found in Robinett and Wilson (2009, 2010b).

The total exergy for the present system is here the system Hamiltonian, given by,

$\mathcal{H}=\mathcal{H}_{m}+\mathcal{H}_{i}>0$

where $\mathcal{H}$ is the system Hamiltonian, $\mathcal{H}_{m}$ is the mechanical exergy, and $\mathcal{H}_{i}$ is the information exergy.

To enable application of (i) a feedforward control force based on wave prediction that seeks wave-by-wave impedance matching using the best available estimates, and (ii) a feedback force to provide closed-loop trajectory tracking, the force $F_{L}(t)$ in Eq. (1) is expressed as $\left(F_{r}\right.$ denoting the feedforward force and $F_{f b}$ the feedback force),

$F_{L}(t)=F_{r}(t)+F_{f b}(t)$

Note that $F_{r}$ and $F_{f b}$ may be applied by different actuators, connected in parallel. Further, parts of $F_{r}$ and $F_{f b}$ may also be applied by different actuators, depending on whether they are resistive or reactive. Letting $M=[m+\bar{a}(\infty)]$ for convenience, the mechanical exergy $\mathcal{H}_{m}$ is defined as, 


$$
\begin{aligned}
\mathcal{H}_{m}(t)= & \frac{1}{2} M\left(v-v_{r}\right)^{2}+\frac{1}{2} k\left(x-x_{r}\right)^{2} \\
& +\int_{-\infty}^{t} \int_{-\infty}^{\infty} h_{a}(\tau)\left[v(\xi-\tau)-v_{r}(\xi-\tau)\right] \mathrm{d} \tau \\
& {\left[v(\xi)-v_{r}(\xi)\right] \mathrm{d} \xi }
\end{aligned}
$$

where the substitutions $\dot{x}=v$, and $\dot{x}_{r}=v_{r}$ may be used. Note that the last term represents an integral from $-\infty$ to the present time $t$ of a reactive force times velocity. The integral of reactive power over a time interval is zero for lossless (ideal) linear actuators, so that $\mathcal{H}_{m}(t)$ as a whole is positive definite.

The information exergy $\mathcal{H}_{i}$ is a function of the parameterestimate uncertainty, and is defined as,

$\mathcal{H}_{i}(t)=\frac{1}{2}\{\phi\}^{T}[\Gamma]^{-1}\{\phi\}+\frac{1}{2} K_{x}\left(x-x_{r}\right)^{2}+\frac{1}{2} K_{a}\left(\dot{v}-\dot{v}_{r}\right)^{2}$

For estimates $\hat{M}, \hat{k}, \hat{c}_{d}, \hat{R}$, and $\hat{F}$, the vector $\phi$ is here defined as

$\{\phi\}^{T}=\left[\hat{M}-M, \hat{k}-k, \hat{c}_{d}+c_{d}, 1 / 2-\hat{F}, 1-\hat{R},-\hat{R}, 2 \hat{c}_{d}\right]$

$K_{x}$ and $K_{a}$ are the feedback gains associated with position and acceleration errors, respectively, relative to the reference trajectory.

The elements of $\{\phi\}$ are thus seen to be related to the parameter estimates.

Further,

$[\Gamma]=\operatorname{diag}\left[\gamma_{1}, \gamma_{2}, \ldots \gamma_{7}\right]$

$[\Gamma]$ is a diagonal matrix whose elements represent the 'adaptation gains' associated with each parameter estimate that is being updated.

The analysis below summarizes the steps leading to the conditions under which $\mathcal{H}$ is a Lyapunov function. Recall that a function associated with the trajectory of a dynamic system is a Lyapunov function if it is positive definite and if its time derivative evaluated over that trajectory is negative definite. The trajectory in motion space over which $\mathcal{H}$ and $d \mathcal{H} / \mathrm{d} t$ are examined here is the 'reference trajectory' given by $\left(x_{r}, v_{r}\right)$. The conditions for $\mathcal{H}$ to be a Lyapunov function also represent the minimum exergy solution where the sum of the mechanical and information exergy is minimized.

The following analysis uses the substitutions $h_{a}=\hat{R} h_{a h}$, $h_{r}=\hat{R} h_{r h}$, and $F_{f}=\hat{F} F_{f h}$ into Eqs. (24) and (1). In addition, Eq. (23) is also substituted into Eq. (1). Differentiation of the total Hamiltonian $\mathcal{H}$ with respect to $t$ leads to an expression for the time derivative $\mathrm{d} \mathcal{H} / \mathrm{d} t$,

$$
\begin{aligned}
\frac{\mathrm{d} \mathcal{H}(t)}{\mathrm{d} t}= & {\left[M \dot{v}+k x-M \dot{v}_{r}-k x_{r}\right.} \\
& \left.+\int_{-\infty}^{\infty} h_{a}(\tau)\left[v(t-\tau)-v_{r}(t-\tau)\right] \mathrm{d} \tau\right]\left(v-v_{r}\right) \\
& +\left[K_{x}\left(x-x_{r}\right)+K_{a}\left(\dot{v}-\dot{v}_{r}\right)\right]\left(v-v_{r}\right) \\
& +\{\dot{\phi}\}^{T}[\Gamma]^{-1}\{\phi\}
\end{aligned}
$$

The dynamic model for heave oscillations can be expressed as [from Eq. (1)],

$$
\begin{aligned}
M \dot{v}+k x=- & c_{d} v-\int_{0}^{\infty} h_{r}(\tau) v(t-\tau) \mathrm{d} \tau \\
& +\int_{-\infty}^{\infty} h_{f}(\tau) \eta\left(x_{B} ; t-\tau\right) \mathrm{d} \tau+F_{r}+F_{f b}
\end{aligned}
$$

Note that $x$ and $v$, respectively, denote the actual heave displacement and velocity of the buoy. The feedforward force $F_{r}$ is specified based on the best available parameter estimates and the best available estimate for the reference trajectory. Thus,

$$
\begin{aligned}
F_{r}(t)= & \hat{M} \dot{v}_{r}+\hat{k} x_{r}-\hat{c}_{d} v_{r}-\int_{-\infty}^{\infty} h_{b h}(\tau) v_{r}(t-\tau) \mathrm{d} \tau \\
& +\int_{-\infty}^{\infty} h_{a h}(\tau) v_{r}(t-\tau) \mathrm{d} \tau
\end{aligned}
$$

The feedback force $F_{f b}$ is specified as,

$F_{f b}(t)=-K_{x}\left(x-x_{r}\right)-K_{b}\left(v-v_{r}\right)-K_{a}\left(\dot{v}-\dot{v}_{r}\right)$

where additional velocity feedback is also introduced along with the associated gain $K_{b}$. An additional integral term (i.e. integral of the displacement error up to the present time) may also be used in feedback. In the presence of an oscillation constraint, $h_{r h}$ in Eq. (29) needs to be replaced by a modified impulse-response function where

$h_{r h c}(t)=\frac{2}{\pi} \int_{0}^{\infty}\left[b_{h}(\omega)+\Lambda(\omega)\right] \cos \omega t \mathrm{~d} \omega$

It should be noted that the oscillation constraint as specified through Eqs. (13), (20), and (21) is applied on the reference velocity. Unless effective tracking performance is achieved, the actual velocity may exceed the specified constraint. For this reason, in this work, an additional term may be introduced into $F_{r}$ as,

$$
\begin{aligned}
F_{r}= & \hat{M} \dot{v}_{r}+\hat{k} x_{r}-\left[\hat{c}_{d}+\alpha_{c} \Lambda_{m}\right] v_{r} \\
& -\int_{-\infty}^{\infty} h_{b h}(\tau) v_{r}(t-\tau) \mathrm{d} \tau \\
& +\int_{-\infty}^{\infty} h_{a h}(\tau) v_{r}(t-\tau) \mathrm{d} \tau
\end{aligned}
$$


where $\Lambda_{m}=\max [\Lambda(\omega)]$, and $\alpha_{c}$ is a constant $\left(\alpha_{c}=0.6\right.$ is used in the present simulations).

Substituting Eq. (29), and Eqs. (30), and (31) into Eq. (28), and further, adding and subtracting $c_{d} v_{r}$ on the right side, the following expression is obtained. Note that the substitution of Eq. (29) ensures that the device dynamics are satisfied. The time derivative of the Hamiltonian $\mathcal{H}$ can now be written as,

$$
\begin{aligned}
\frac{\mathrm{d} \mathcal{H}(t)}{\mathrm{d} t}= & {\left[-c_{d} v-\int_{0}^{\infty} h_{r}(\tau) v(t-\tau) \mathrm{d} \tau\right.} \\
& \left.+\int_{-\infty}^{\infty} h_{f}(\tau) \eta\left(x_{B} ; t-\tau\right) \mathrm{d} \tau\right]\left(v-v_{r}\right) \\
& +\left[\hat{M} \dot{v}_{r}+\hat{k} x_{r}-\hat{c}_{d} v_{r}\right. \\
& \left.-\int_{-\infty}^{\infty} h_{b h}(\tau) v_{r}(t-\tau) \mathrm{d} \tau\right]\left(v-v_{r}\right) \\
& +\left[\int_{-\infty}^{\infty} h_{a h}(\tau) v_{r}(t-\tau) \mathrm{d} \tau\right]\left(v-v_{r}\right) \\
& -\left[K_{x}\left(x-x_{r}\right)-K_{a}\left(\dot{v}-\dot{v}_{r}\right)-K_{b}\left(v-v_{r}\right)\right. \\
& \left.-M \dot{v}_{r}-k x_{r}\right]\left(v-v_{r}\right) \\
& +\left[\int_{-\infty}^{\infty} h_{a}(\tau)\left[v(t-\tau)-v_{r}(t-\tau)\right] \mathrm{d} \tau\right]\left(v-v_{r}\right) \\
& +\left[K_{x}\left(x-x_{r}\right)+K_{a}\left(\dot{v}-\dot{v}_{r}\right)\right]\left(v-v_{r}\right) \\
& +\{\dot{\phi}\}^{T}[\Gamma]^{-1}\{\phi\}
\end{aligned}
$$

Some simplification leads to,

$$
\begin{aligned}
\frac{\mathrm{d} \mathcal{H}}{\mathrm{d} t}= & {\left[-c_{d}\left(v-v_{r}\right)+(\hat{M}-M) \dot{v}_{r}+(\hat{k}-k) x_{r}\right.} \\
& -\left(c_{d}+\hat{c}_{d}\right) v_{r} \\
& \left.-\int_{-\infty}^{\infty} h_{a}(\tau) v(t-\tau) \mathrm{d} \tau\right]\left(v-v_{r}\right) \\
& +\left[-\int_{-\infty}^{\infty} h_{b}(\tau) v(t-\tau) \mathrm{d} \tau\right. \\
& +\int_{-\infty}^{\infty} h_{f}(\tau) \eta\left(x_{B} ; t-\tau\right) \mathrm{d} \tau \\
& \left.-\int_{-\infty}^{\infty} h_{b h}(\tau) v_{r}(t-\tau) \mathrm{d} \tau\right]\left(v-v_{r}\right) \\
& +\left[\int_{-\infty}^{\infty} h_{a h}(\tau) v_{r}(t-\tau) \mathrm{d} \tau-K_{b}\left(v-v_{r}\right)\right. \\
& \left.+\int_{-\infty}^{\infty} h_{a}(\tau)\left[v(t-\tau)-v_{r}(t-\tau)\right] \mathrm{d} \tau\right]\left(v-v_{r}\right) \\
& +\{\dot{\phi}\}^{T}[\Gamma]^{-1}\{\phi\}
\end{aligned}
$$

Next using Eqs. (53), (60), and the relationship,

$$
\begin{gathered}
2 \int_{-\infty}^{\infty} h_{b h}(\tau) v_{r}(t-\tau) \mathrm{d} \tau+\hat{c}_{d} v_{r} \\
=\int_{-\infty}^{\infty} h_{f h}(\tau) \eta\left(x_{B} ; t-\tau\right) \mathrm{d} \tau
\end{gathered}
$$

$$
\begin{aligned}
\frac{\mathrm{d} \mathcal{H}}{\mathrm{d} t}= & {\left[-c_{d}\left(v-v_{r}\right)+(\hat{M}-M) \dot{v}_{r}+(\hat{k}-k) x_{r}\right.} \\
& \left.-\left(c_{d}+\hat{c}_{d}\right) v_{r}-K_{b}\left(v-v_{r}\right)\right]\left(v-v_{r}\right) \\
& +\left[-\hat{R} \int_{-\infty}^{\infty} h_{a h}(\tau)\left[v(t-\tau)-v_{r}(t-\tau)\right] \mathrm{d} \tau\right. \\
& \left.-\left(\frac{1}{2}-\hat{F}\right) \times \int_{-\infty}^{\infty} h_{f h}(\tau) \eta\left(x_{B} ; t-\tau\right) \mathrm{d} \tau\right]\left(v-v_{r}\right) \\
& +\left[\int_{-\infty}^{\infty} h_{a h}(\tau) v_{r}(t-\tau) \mathrm{d} \tau\right. \\
& \left.-\hat{R} \int_{0}^{\infty} h_{r h}(\tau) v_{r}(t-\tau) \mathrm{d} \tau+\hat{c}_{d} v_{r}\right]\left(v-v_{r}\right) \\
& +\{\dot{\phi}\}^{T}[\Gamma]^{-1}\{\phi\} .
\end{aligned}
$$

With $\{\phi\}$ defined as shown in Eq. (26), $[\Gamma]$ expressed as in Eq. (27), and some algebra, it can be shown that

$$
\begin{aligned}
\frac{\mathrm{d} \mathcal{H}}{\mathrm{d} t}=- & c_{d}\left(v-v_{r}\right)^{2}-K_{b}\left(v-v_{r}\right)^{2}-\int_{-\infty}^{\infty} h_{b}(\tau)[v(t-\tau) \\
& \left.-v_{r}(t-\tau)\right] \mathrm{d} \tau\left[v(t)-v_{r}(t)\right]
\end{aligned}
$$

if the parameter estimates are updated according to,

$$
\begin{aligned}
& \dot{\hat{M}}=-\gamma_{1} \dot{v}_{r}\left(v-v_{r}\right) \\
& \dot{\hat{c}} d=-\gamma_{2} v_{r}\left(v-v_{r}\right) \\
& \dot{\hat{k}}=-\gamma_{3} x_{r}\left(v-v_{r}\right) \\
& \dot{\hat{F}}=2 \gamma_{4}\left(\int_{-\infty}^{\infty} h_{f h}(\tau) \eta\left(x_{B} ; t-\tau\right) \mathrm{d} \tau\right)\left(v-v_{r}\right) \\
& \dot{\hat{R}}=\gamma_{5}\left(\int_{-\infty}^{\infty} h_{a h}(\tau) v_{r}(t-\tau) \mathrm{d} \tau\right)\left(v-v_{r}\right)
\end{aligned}
$$

The equalities in Eq. (39) are obtained by collecting like terms associated with estimate uncertainties (e.g. $(\hat{M}-M)$, $(\hat{k}-k)$, etc). Note further that the estimates $\hat{M}, \hat{K}, \hat{R}$, etc are slowly varying with time, while the exact quantities $M$, $k, R$, etc. are not functions of time. Two other equations are obtained, which for the purpose of estimate updating can be considered redundant without affecting Eq. (38). Of particular note is that the update equations for $\hat{F}$ and $\hat{R}$ require wave and/or oscillation prediction, since both $h_{f h}$ and $h_{a h}$ are non-causal, as discussed in Sect. 3 .

$c_{d}>0, K_{b}$ can be chosen to be $>0$, and $h_{b}(t)$ is an even function of $t$. Although the position coordinate does not appear in Eq. (38), the negative definiteness of $d \mathcal{H} / \mathrm{d} t$ can be shown following the general analysis steps in Robinett and Wilson (2010a). Since $\mathcal{H}>0$ by definition, with $d \mathcal{H} / \mathrm{d} t<$ 0 , the trajectory tracking errors and the parameter estimate errors lie on a stable manifold and convergence is achieved as $t \rightarrow \infty$. Nevertheless, it would be helpful to carry out a closer study of the rate of convergence and the associated stability bounds in further work on the present technique. 
The feedback gains would typically be designed to maximize trajectory tracking performance in test trajectories such as unit steps or pure sinusoids [Robinett and Wilson (2007)]. Standard transient response or frequency-response design techniques may be used, and during this design process, perfect knowledge of the model parameters may be assumed. A more ad hoc procedure is followed in this work, since the equation of motion is a second-order integro-differential equation and since it was desired to maintain that structure (rather than use a state-space approximation for the radiation force convolution term) for a full implementation of wave-by-wave impedance match. The adaptation gains $\gamma_{i}$ are prescribed so as to avoid sudden changes in parameter estimates, and adjustments are made iteratively so that the mean converted power is maximized.

The instantaneous absorbed power is defined as the product of the total instantaneous force applied by the actuator and the actual instantaneous velocity. The applied force $F_{L}(t)$ is,

$$
F_{L}(t)=F_{r}(t)+F_{f b}(t)
$$

The net time-averaged absorbed power $P_{w}$ over the interval $[0, T]$ can be found using,

$P_{w}=\frac{1}{T} \int_{0}^{T}\left[F_{L}(t) v(t)\right] \mathrm{d} t$

The wave power (in $\mathrm{kW}$ ) incident over the device diameter $2 R$ is given by,

$P_{\text {inc }}=0.49 H_{s}^{2} T_{e}(2 R)$

where $H_{s}$ is the significant wave height and $T_{e}$ is the energy period representing the incident irregular wave record. With $P_{w}$ and $P_{i n c}$ expressed in consistent units, the power capture width ratio (or capture factor) was computed using

$\ell=\frac{P_{w}}{P_{\text {inc }}}$

\section{Calculations and results}

Calculations and control simulations were carried out for the cylindrical buoy shown in Fig. 1 . The radius $R_{S}$ was chosen to be $4 \mathrm{~m}$, leading to a draft $D_{r}=2 \mathrm{~m}$. The freeboard was assumed to be $2 \mathrm{~m}$ in these calculations. As mentioned, heave motion was assumed to be predominant, and actuators with linear response and ideal or lossless behavior were assumed for the results with perfect knowledge.

Note that two or more actuators could be used here so long as the required force variations in Sect. 4 are applied on the buoy. In particular, the reactive force component could be applied by an actuator especially designed to match the instantaneous power requirements, while the resistive part of the load could be applied by a different actuator.

In these simulations, the actual device behavior (as opposed to the estimated model) was modeled by an integrodifferential equation using exact parameter values. The exact hydrodynamic coefficients and exciting force amplitude and phase here were computed using the numerical code HYDRAN (HYDRAN (2012)), and were drawn from Korde and Ertekin (2015). The exact linearized viscous damping coefficient was computed using an assumed constant formfriction coefficient over the the entire immersed surface area.

The procedure was programmed in the software environment Matlab. Wave prediction for the given wave conditions (i.e. energy period and significant wave height) for the required duration was obtained using a separate script and read into the adaptive control simulation routine. However, given the close match between the prediction and computed values in Korde (2015) in most simulations, it was decided to use the computed wave records for convenience and speed of execution. Unidirectional sea-states were assumed. PiersonMoskowitz type 2-parameter spectra representing the chosen $H_{s}$ and $T_{e}$ were used, as defined by [e.g. see Falcao (2008)] as,

$S(\omega)=131.5 \frac{H_{s}^{2}}{T_{e}^{4} \omega^{5}} \exp \left[-1054 /\left(T_{e} \omega\right)^{4}\right]$

where $S(\omega)$ represents the power spectral density for a wave frequency $\omega$.

$\eta\left(x_{A} ; t\right)=\sum_{n=1}^{N} \Re\left\{A\left(\omega_{n}\right) \exp \left[-i\left(k\left(\omega_{n}\right) x_{A}-\omega_{n} t+\theta_{n}\right)\right]\right\}$

where,

$A\left(\omega_{n}\right)=\sqrt{2 S\left(\omega_{n}\right) \Delta \omega}$

The phase of the $n$th frequency component, $\theta_{n}$, is a random number between $[0,2 \pi]$.

Results are arranged as follows: Fig. 2 shows the impulseresponse functions for the exciting force (numerical computation compared with analytical approximation). Figure 3 compares the approximate radiation impulse-response function (59) with the 'exact' function based on numerical computation. The next few results are shown for a sea state with $H_{s}=1 \mathrm{~m}$, and $T_{e}=9 \mathrm{~s}$. Figure 4 plots the reference heave velocity found using the approximate parameter estimates and Eq. (16) alongside the actual velocity in heave. Figures 7, 8, and 9 plot the parameter-estimate updates through the simulation. The estimates for the parameters $\hat{F}$ 


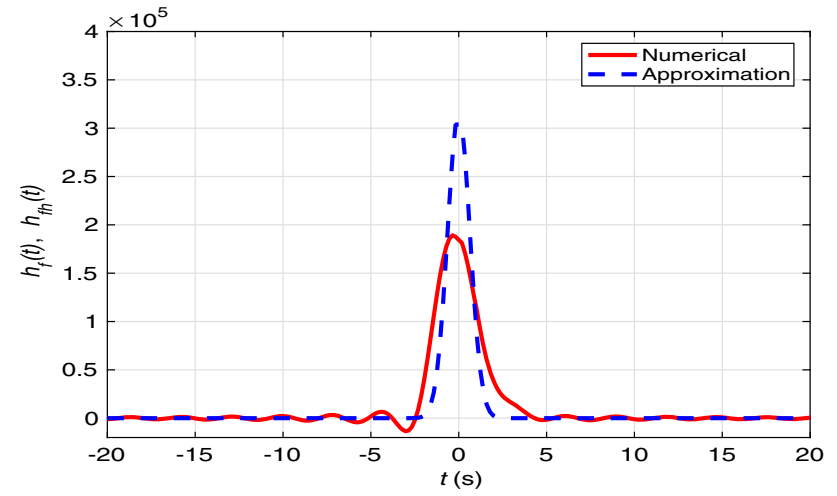

Fig. 2 Exciting force impulse-response function for the buoy in heave. The dashed line in the figure represents an approximation based on the assumption that scattering is negligible and so the Froude-Krylov force component dominates. The solid line shows the more exact variation with the scattering effects included, and is based on results obtained using a Boundary Element computational code

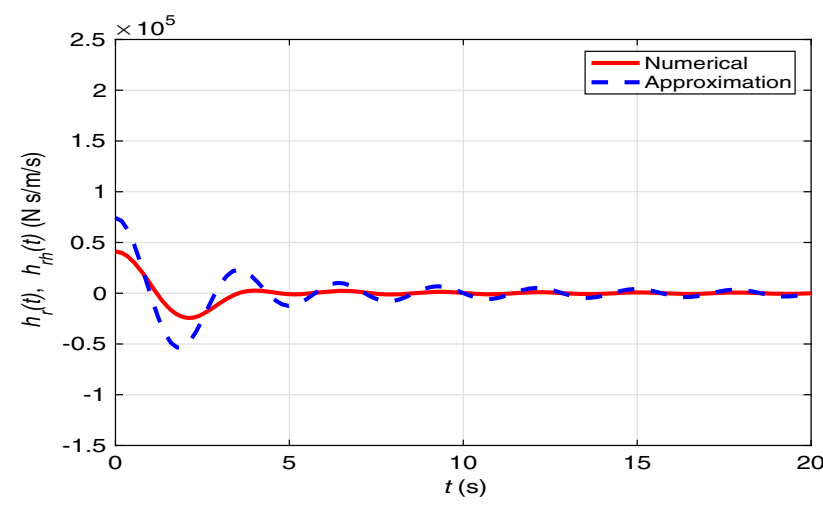

Fig. 3 The radiation impulse-response function for the buoy in heave. The dashed line in the figure represents an approximation based on the assumption that scattering is negligible and so the Froude-Krylov force component dominates. The Haskind-Hanaoka relation is applied on this approximation to obtain radiation damping variation in the frequency domain. The time-domain impulse-response function is found via inverse cosine transformation of the radiation damping. The solid line shows the more exact variation with the scattering effects included, and requires a Boundary Element computational code

and $\hat{R}$ are shown in Figs. 10 and 11, respectively. Fig. 13 compares the forces $F_{b}$ and $F_{r}$ with $F_{f}$. Figure 14 plots the power absorbed by the actuator during the simulation interval. Finally, Fig. 15 plots the capture width ratio variation with energy period based on these simulations. Also provided for comparison are the capture width ratio variations under near-optimal control with perfect knowledge, with imperfect $\hat{M}, \hat{c}_{d}$, and $\hat{k}$ but exact $h_{r}$ and $h_{f}$, and with pure resistive loading, where the damping is set to a constant value approximately exceeding the radiation damping at the peak frequency and no real-time control is attempted.

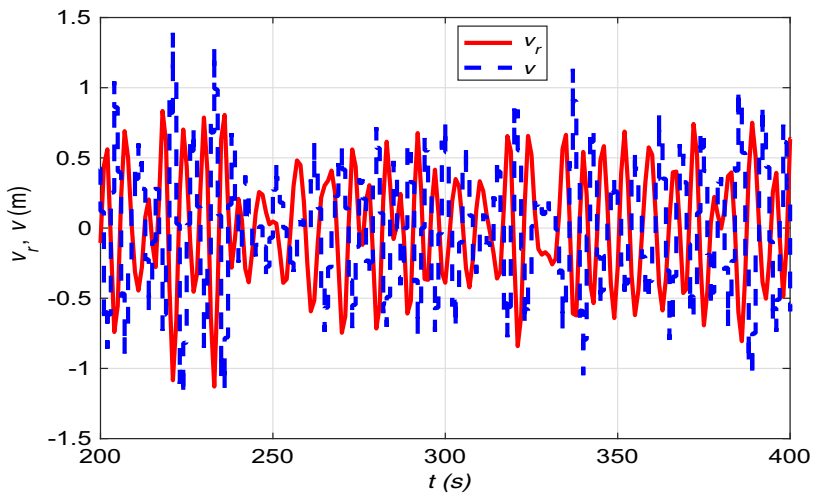

Fig. 4 Reference velocity signal based on approximate parameters compared with the actual velocity. The reference velocity is computed using wave prediction, and is the hydrodynamic velocity optimum under a specified oscillation constraint. The best available estimates for the exciting force impulse-response function, the radiation force impulseresponse function, and the linearized viscous damping are used in this calculation. The actual velocity is the instantaneous heave velocity of the buoy as determined by Eq. (1). The irregular wave input here is generated for $H_{s}=1 \mathrm{~m}$ and $T_{e}=9 \mathrm{~s}$

\section{Discussion of results}

This work studies wave-by-wave impedance matching control in irregular waves based on wave prediction, where the wave prediction is based on an up-wave measurement and uses a deterministic model that accounts for a realistic groupvelocity range and assumes linear propagation. Such control was discussed, for instance, in Korde (2015), where all parameters involved in the device dynamic response are assumed to be perfectly known. In practice, however, device parameters may be imperfectly known, affecting the accuracy of the control forces. The goal of the present paper is to develop a strategy that will approximate the desired control while minimizing the detrimental effect of imperfect parameter knowledge. The method relies on feedforward and feedback forces to enable close tracking of the hydrodynamic velocity optimum and parameter estimate updating. Oscillation constraints are applied within the formulation using an approximate procedure based on the frequency-domain approach of Evans (1981) [a more direct form of the approach was used in Korde (2015)]. Both optimum trajectory and feedforward force are evaluated using the best available current estimates for the parameters, and are updated each time the estimates are updated. Figure 2 compares the approximate exciting force impulse-response function used in the simulations with the exact exciting force impulse-response function based on a numerical code. The approximate function based on the small-body Froude-Krylov force approximation overpredicts the impulse-response function, but has a shorter oscillatory signature (than the more exact function) in the $t<0$ range. Figure 3 compares the approximate and more exact radiation impulse-response functions. The approxi- 


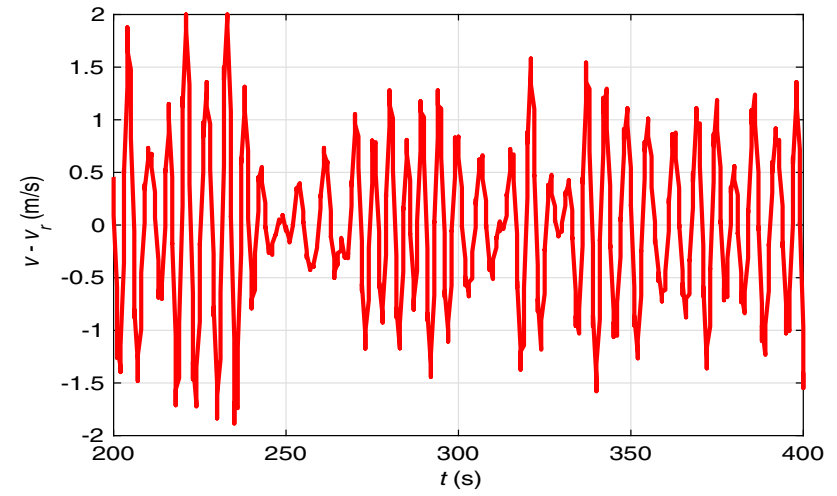

Fig. 5 Plot showing the instantaneous racking error for velocity; i.e. the difference between actual velocity and the reference velocity. This plot shows the difference between the actual and reference velocities appearing in Fig. 4. The irregular wave input here is generated for $H_{s}=$ $1 \mathrm{~m}$ and $T_{e}=9 \mathrm{~s}$

mate function is again found to have a greater magnitude, and there is greater oscillation in the approximate function. These differences could potentially cause inaccuracies in the estimated reference trajectory. The two observations are understandable, however, in that for the floating vertical cylinder geometry the diffraction force subtracts from the Froude-Krylov force in the low-frequency range, and becomes comparable at higher frequencies. Truncation time for both is seen to be in the 20-30 s range.

Figure 4 shows that differences in the reference velocity and actual velocity persist throughout the simulation interval. Given the approximations embedded in the impulse-response functions $h_{a}$ and $h_{b}$ as well as the other parameters $\hat{M}, \hat{c}_{d}$, and $\hat{k}$, an exact match probably cannot be expected. However, since the reference velocity itself is based on the approximate impulse-response function $h_{o h}$, it is perhaps not desirable to seek too precise a match relative to this approximate reference velocity. Figure 5 plots the instantaneous tracking error for velocity. Tracking errors are seen to be comparable in magnitude to the actual velocities for the case shown. Greater deviations are observed between the reference oscillation and the actual oscillation of Fig. 6. It should be noted that, the reference oscillation in this work is computed using numerical integration of the reference velocity signal with a first-order trapezoidal integration procedure. Therefore, any small error in the velocity determination can potentially increasingly add to errors in the oscillation signals. Further, both reference and actual oscillations are seen to be within the specified oscillation constrains $x_{r}(t), x(t) \leq D_{r} . D_{r}=2 \mathrm{~m}$ here. It is recalled that the oscillation constraint is not a 'hard' constraint and is here imposed through addition of damping. In a practical implementation, real-time measurements of the actual velocity and displacement would be shown in the plots in Figs. 4 and 6 and used in generating the feedback force

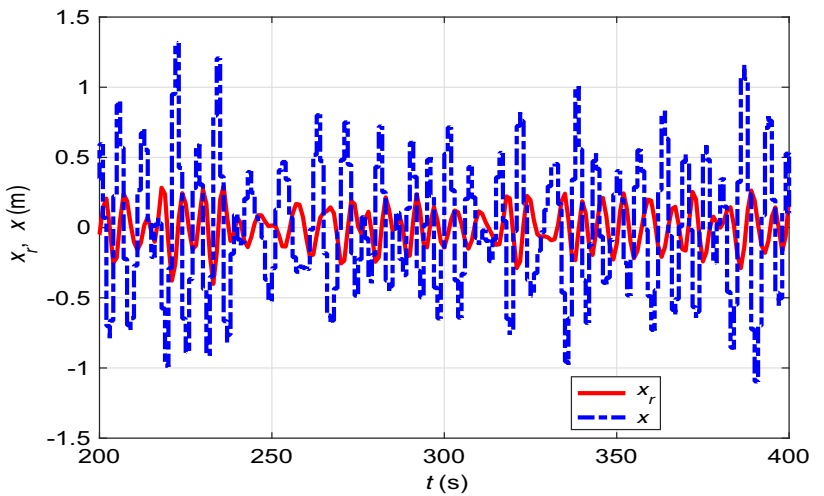

Fig. 6 Reference oscillation displacement based on numerical integration of reference velocity signal, compared with actual oscillation. The reference velocity is based on approximate parameters. The reference velocity 'estimate' is computed using wave prediction, and is the hydrodynamic velocity optimum 'estimate' under a specified oscillation constraint. The best available estimates for the exciting force impulseresponse function, the radiation force impulse-response function, and the linearized viscous damping are used in this calculation. In practice, the actual velocity and displacement would be measured, but here they are found through simulation. The irregular wave input here is generated for $H_{s}=1 \mathrm{~m}$ and $T_{e}=9 \mathrm{~s}$

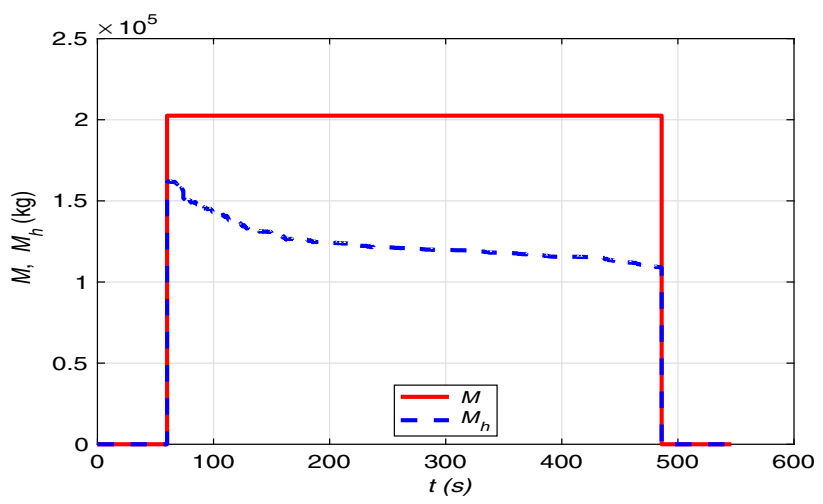

Fig. 7 Plot showing how the estimates $\hat{M}$ for $M$ evolve through the procedure. The evolution is given by the first of Eq. (39). The irregular wave input is generated for $H_{s}=1 \mathrm{~m}$, and $T_{e}=9 \mathrm{~s}$

$F_{f b}$. As already mentioned, in this work, the actual velocity and displacement are obtained via simulation.

Note that parameters $\hat{M}, \hat{c}_{d}$, and $\hat{k}$ are updated at intervals of $N_{u}$ time steps, and in the simulations here, $N_{u}=10$ was chosen, corresponding to a 10-times slower estimate variation. Shown in the following figures are the successive estimates through the simulation interval and the exact values (which are known here but likely unavailable in practice). Figure 7 shows the evolution of the estimate $\hat{M}$ for $m+a(\infty)$. The change in $\hat{M}$ with respect to time is negative throughout, and becomes smaller beyond $t=300$, showing that gradual convergence is achieved, albeit with greater error than for the $\hat{c}_{d}$ and $\hat{k}$ estimates. $\hat{c}_{d}$ and $\hat{k}$ converge to their desired values more closely, as seen in Figs. 8 and 9, respectively. The initial estimates for the parameters 


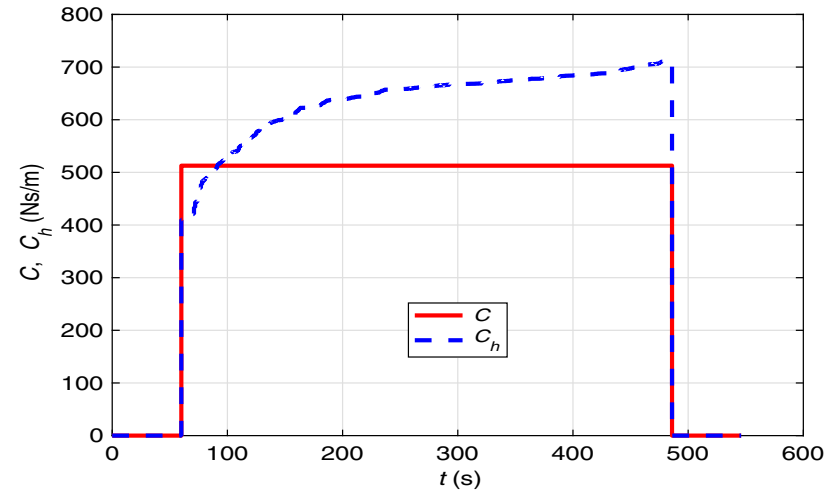

Fig. 8 Plot showing how the estimates $\hat{c}_{d}$ for $c_{d}$ evolve through the procedure. This evolution is given by the second of Eqs. (39). The irregular wave input is generated for $H_{s}=1 \mathrm{~m}$, and $T_{e}=9 \mathrm{~s}$

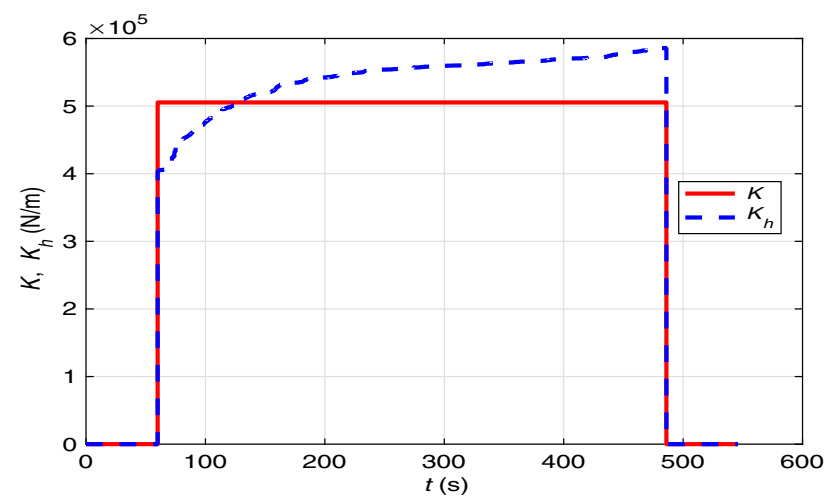

Fig. 9 Plot showing how the estimates $\hat{k}$ for $k$ evolve through the procedure. This evolution is given by the third of Eq. (39). The irregular wave input is generated for $H_{s}=1 \mathrm{~m}$, and $T_{e}=9 \mathrm{~s}$

are assumed to be about $80-120 \%$ of their known exact values, although convergence is also observed in simulations for different combinations of initial estimates. However, some a priori knowledge as to whether the initial estimates are smaller or greater than the exact values may be desirable in practice, and could be utilized in choosing the signs of the adaptation gains. Note that the update relations for $\hat{M}, \hat{c}_{d}$, and $\hat{K}$ are all first-order differential equations. Further, estimates are updated at intervals of 10 time increments, so that considerable 'averaging' of the trajectory errors is allowed. Therefore, a largely monotonic behavior is what would be expected for these estimates. Note, however, that the adaptation procedure attempts to optimize the overall performance index $\mathcal{H}$ without separately penalizing errors in evolving parameter estimates of particular parameters. Figures 10 and 11 show a less monotonic variation for $\hat{F}$ and $\hat{R}$. Moreover, these variations appear to be more sensitive to the adaptation gain values used in update procedure of Eq. (39). The overall procedure is particularly sensitive to the estimates $\hat{F}$ and $\hat{R}$, which determine the estimated reference trajectory. $\hat{R}$ additionally also determines the feedforward force $F_{r}$. The

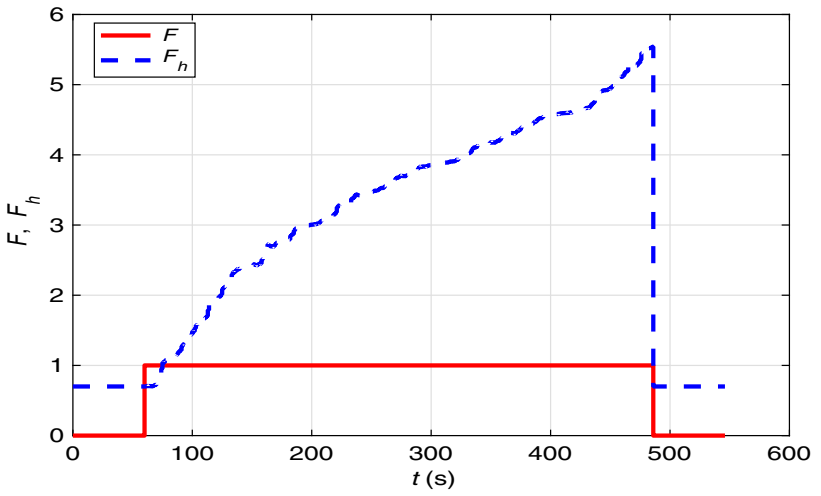

Fig. 10 Plot showing how the estimates $\hat{F}$ for $F$ evolve through the procedure. This evolution is given by the fourth of Eq. (39). Note that exact match would imply $\hat{F}=1$. The irregular wave input is generated for $H_{s}=1 \mathrm{~m}$, and $T_{e}=9 \mathrm{~s}$

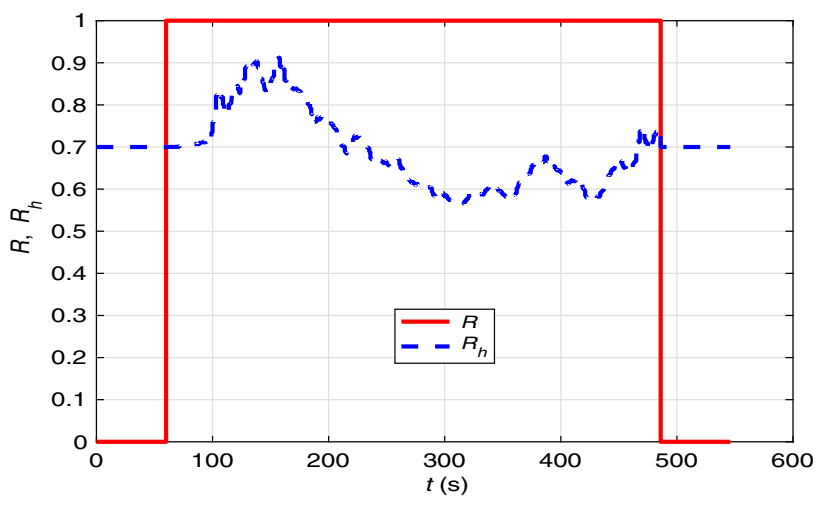

Fig. 11 Plot showing how the estimates $\hat{R}$ for $R$ evolve through the procedure. This evolution is given by the last of Eq. (39). Note that exact match implies $\hat{R}=1$. The irregular wave input is generated for $H_{s}=1 \mathrm{~m}$, and $T_{e}=9 \mathrm{~s}$

'steady-state errors' seen in the case of $\hat{R}$ and $\hat{F}$ are much greater than for $\hat{M}, \hat{c}_{d}$, and $\hat{k}$, which is a possible indication that one-parameter, multiplicative uncertainty models are perhaps insufficient for the impulse-response variations at hand. It should also be noted that the presence of the ratio $\hat{R} / \hat{F}$ in the update relation for the reference trajectory [Eq. (18)] makes the trajectory update oversensitive to small changes in $\hat{F}$. Further work on two or three parameter representations (additive and multiplicative) for the impulseresponse functions and trajectory update relations may be warranted, and may provide better results. The stability margin indicated by Eq. (38) also needs to be examined further in a more formal treatment.

Figure 12 plots the total instantaneous applied force $F_{L}$ (" $F_{c l}$ ") together with the instantaneous heave velocity of the buoy. $F_{L}$ represents the total load applied on the heave oscillation. The phase difference between $F_{L}$ shows the presence of a reactive component in $F_{L}$.

Figure 13 plots the variation of the forces that need to be applied by the actuators to provide the control under dis- 


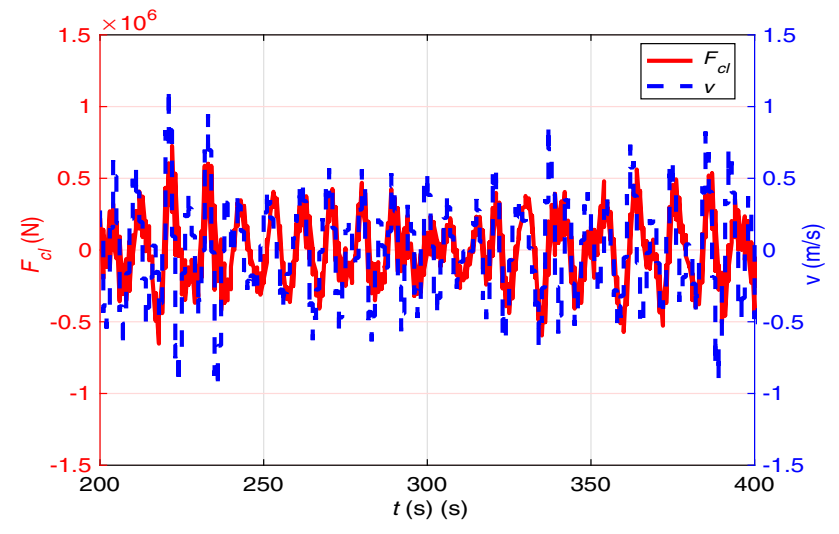

Fig. 12 The instantaneous total applied force on the buoy along with the instantaneous velocity. This force represents the load applied on the buoy. The irregular wave input is generated for $H_{s}=1 \mathrm{~m}$, and $T_{e}=9$ s

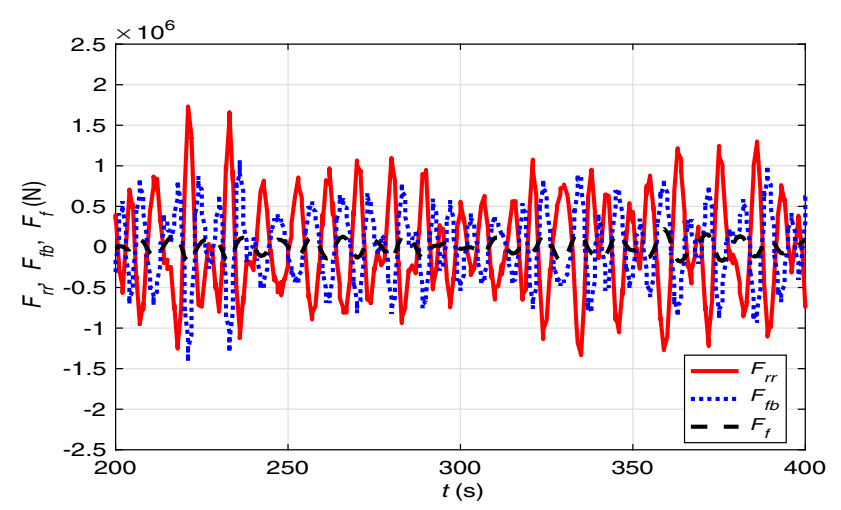

Fig. 13 Plot comparing the instantaneous forces to be applied by the actuators with the instantaneous exciting force during the simulation interval. Note that the feedforward force $F_{r}$ appears to be significant, exceeding the exciting force at some instants. Even though the oscillations are constrained in this work, forces are not. The irregular wave input is generated for $H_{s}=1 \mathrm{~m}$, and $T_{e}=9 \mathrm{~s}$

cussion. Both the feedback force $F_{b}$ and the feedforward force $F_{r}$ are plotted, and shown alongside for comparison is the exciting force $F_{f}$. Note that this is the exact exciting force variation computed using the exact $h_{f}$. It is seen that the feedback force $F_{b}$ is here smaller than the feedforward force $F_{r}$. However, the mean-square $F_{r}$ here appears to exceed the mean-square $F_{f}$. The large feedforward force requirement can be understood in relation to buoy size and needs to be weighed against the power conversion gains available with this approach. In particular, here, a relatively small buoy $(R=4 \mathrm{~m})$ is to be forced to oscillate close to the desired optimum velocity in much longer waves. Device size selection must therefore be informed by available actuator technology. Further, the force magnitudes observed in plots such as Fig. 13 need to be taken into account in actuator selection/design. It appears that sharing this force over multiple low-dissipation actuators may be an alternative worth considering further. Additionally, once the actuators are chosen, suitable force constraints (in addition to oscillation constraints) should be incorporated into the procedure (e.g. Bacelli and Ringwood 2013).

Figure 14 shows the instantaneous net absorbed power variation over the simulation interval. Some intervals of 'negative power absorption' are observed in the plot, suggesting considerable deviations relative to the exact hydrodynamic velocity optimum and the corresponding load. Not surprisingly, therefore, the use of approximate parameter estimates has resulted in a noticeable loss of power capture.

Figure 15 compares the power capture factor for a range of wave conditions. Two-parameter spectra for a range of $T_{e}$ values but with $H_{S}=1 \mathrm{~m}$ were used to generate the irregular wave inputs to these simulations. It should be pointed

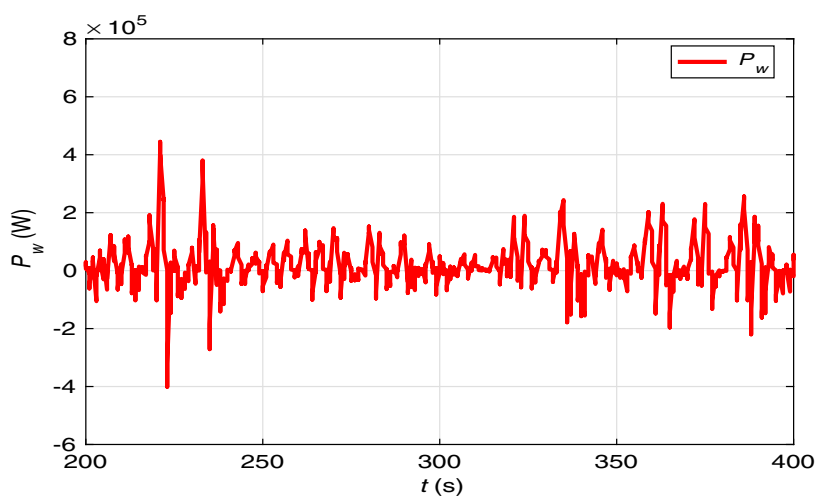

Fig. 14 The variation of instantaneous net absorbed power under the present adaptive control scheme, as given by $P_{w L}(t)-P_{w f}(t)$. Note that power is returned at some instants, and perfect absorption is not possible through the simulation period owing to the mismatch between parameter estimates and the exact values. The irregular wave input is that generated for $H_{s}=1 \mathrm{~m}$, and $T_{e}=9 \mathrm{~s}$

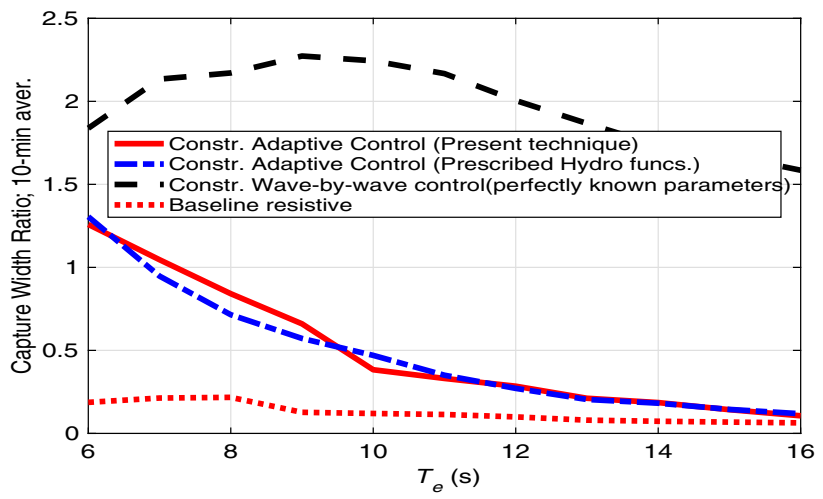

Fig. 15 Power capture factor ('capture width ratio') for a range of irregular wave conditions, defined by spectral energy period values $T_{e}$. Shown for comparison are capture factor ratios when perfect knowledge of parameters is available, when perfect knowledge of the hydrodynamic impulse-response functions is assumed, and also when resistive control alone is present. Oscillations are constrained to be $\leq D_{r}$ (i.e. the buoy draft) 
out that the capture width ratios with approximate parameters and adaptive estimation are noticeably smaller than those available with near-optimal control where exact knowledge to all parameters and hydrodynamic impulse-response functions was available. Note that capture width ratios greater than 1 imply that more energy than directly incident over the buoy diameter is being converted. This situation is made possible by the so-called 'antenna effect' associated with small axisymmetric heaving body converters, where capture width ratios approaching $\lambda /(2 \pi D)$ may be achieved with a heaving buoy of diameter $D$, with $\lambda$ representing the wave length (providing this can be achieved without exceeding the swept-volume constraint). Also interesting to note is the small difference between the two cases when the hydrodynamic impulse-response functions are approximately known and updated in the procedure, and when the hydrodynamic impulse-response functions are known exactly and not updated. In some sea-states, the full adaptive procedure performs slightly better. It is likely that having a greater number of parameters to 'adapt on' allows the adaptation procedure to optimize the performance index better. However, this conjecture needs to be verified with further work. The time-averaged capture width ratio here is seen to decrease as the long-wave content in the wave input grows (increasing $T_{e}$ ). Near-optimum power capture requires large oscillations for the given device size $(R=4) \mathrm{m}$ in swelldominated wave fields. The swept volume constraint restricts the maximum oscillations to the device draft $D_{r}$, which, given the relatively small radiation damping for small $k R$ ( $k R \sim 0.11$ for $T_{e}=12 \mathrm{~s}$ ) for the present, vertical cylinder, geometry, limits the achievable power capture. Note that the power capture width ratios are still appreciably greater than those found for the case with constant damping.

There is considerable room for optimization available in the choice of the feedback gains and the adaptation gains, and further improvements in power capture should follow, as a design strategy for gain selection evolves. In addition, it may be worth examining approaches to incorporate referencetrajectory determination into the overall formulation, by adding an absorbed-power type performance function to the system Hamiltonian in Eq. (22). Note that a number of recently discussed approaches in the literature determine the 'optimal' trajectory based on an absorbed-energy type performance criterion rather than a priori specifying the hydrodynamic velocity optimum as the optimal trajectory [see for instance, Cretel et al. (2011), Fusco and Ringwood (2014)]. In the absence of oscillation constraints, the two approaches should lead to equivalent results. In the presence of oscillation constraints, the performance-index based approach may allow a better use of the available 'oscillation space' under constraints. In addition, such an approach would also enable a more seamless integration of trajectory updates within the procedure. Finally, it should be pointed out that improvement may also be desirable in the integration approach used in the present simulations. It is likely that better accuracy will reduce the build-up of 'phase errors' in the calculation of instantaneous displacement and velocity, affecting the 'phase matches' between the load component $F_{L}(t)$ and velocity $v(t)$. Although the performance is modest when compared against the performance available with exact parameter knowledge, the present work constitutes a first-step effort, and it appears worth proposing that, further performance improvements would be possible for the case with approximate knowledge of device parameters and hydrodynamic impulse-response functions, if an improved adaptive estimation and control strategy derived from the present exergy-based approach were to be adopted in a practical implementation.

\section{Conclusion}

Prior results on wave-by-wave impedance matching control based on deterministic wave prediction have assumed exact knowledge of device parameters such as in-air mass, hydrostatic stiffness, viscous damping, and the hydrodynamic force kernels (i.e. radiation impulse-response function and exciting force impulse-response function). Since exact knowledge of these quantities is frequently unavailable, this work examined an approach to reducing the loss of performance arising from uncertainties in parameter knowledge. This was attempted by seeking to track the best estimated reference trajectory (representing the hydrodynamic velocity optimum under prescribed oscillation constraints) while improving the available parameter estimates. The overall formulation for trajectory tracking and parameter estimation was derived using a Lyapunov function based on the system Hamiltonian formed by combining the mechanical exergy and the information exergy. The instantaneous mechanical exergy was formed by adding the kinetic and potential energies for the device, while the information exergy was expressed in terms of the parameter estimate errors and trajectory tracking error. Since the overall Hamiltonian for the system was positive definite, negative definiteness of its time derivative was required for the trajectory tracking and parameter estimate errors asymptotically to be driven to zero. Both feedforward and feedback forces were used. Oscillations were constrained to be less than the device draft via a 'soft' constraint (i.e. not involving end-stops or other hardware-imposed inequalities). A predominantly heaving cylindrical buoy was used. Draft was assumed to equal freeboard for constraint application. Power absorption was relative to a deeply submerged reaction mass assumed stationary relative to the sea floor. The computation of the feedforward force and the parameter estimate update conditions required wave prediction. In this work, uni-directional waves were assumed, and determinis- 
tic prediction was assumed to be available [for instance, as illustrated in Korde (2015)].

Simulations were carried out in irregular wave conditions derived from 2-parameter spectra over a range of energy periods. Simulation results examined trajectory tracking and parameter estimate update performance as well as power capture. The present work represents a first step towards a fuller implementation. It was observed that the present procedure did lead to capture width ratios greater than those available with resistive control under 'swept volume' oscillation constraints. The present results appear to suggest that the present single-parameter, multiplicative uncertainty model for radiation and exciting force impulse-response functions may not be sufficient, and a better approach may be worth considering. Since perfect knowledge of mechanical parameters and device hydrodynamics is rarely available (e.g. often assumptions are implicit in the determination of hydrostatic stiffness, linearized viscous damping, and the radiation and diffraction forces), a procedure such as examined here would be desirable in practical implementations. The present work considered a single-mode vertical cylinder buoy. However, the method studied here may be applied to other single-mode devices as well. Further work to examine improvement of any wave prediction inaccuracies and incorporation of measurement errors would also be beneficial. In addition, extensions to other geometries and multiple mode oscillations could be worth considering in future work.

Acknowledgments The authors would like to thank Professor R. Cengiz Ertekin for providing the added mass, radiation damping, and exciting force variations used in this work. UAK would like to acknowledge Larry and Linda Pearson and the SDSM\&T Foundation for support toward this work through the Pearson endowment.

\section{Appendix}

The steps leading to the approximate impulse-response functions for the exciting force and radiation force are discussed below.

For a heaving cylinder with $R_{s}=4 \mathrm{~m}$, here, $D_{r}=$ $R_{s} / 2=2 \mathrm{~m}$, so that $k D_{r} \approx 0.21$ or $k R \approx 0.4$ for a wavelength $\lambda_{w}=60 \mathrm{~m}$, for which the deep-water wave period $T_{w}=6.2 \mathrm{~s}$. For this and longer wave periods, diffraction effects for the present geometry may be small enough to be ignored, so that a Froude-Krylov assumption may be reasonable (see Mavrakos and McIver 1997). Given the interest here in seeking approximations, the Froude-Krylov force is further approximated here to ignore the wave profile variation over the buoy diameter, letting $\mathrm{e}^{-k r} \approx 1$.

$$
F_{f}(i \omega) \approx F_{f k}(i \omega)=\rho g \pi R_{s}^{2} \mathrm{e}^{-k(\omega) D_{r}} A(\omega)
$$

The frequency-response function corresponding to $F_{f}(i \omega)$ above can simply be expressed as,

$H_{f}(i \omega)=F_{f}(i \omega) / A(\omega)$

In deep water, the dispersion relation gives, $k(\omega)=\omega^{2} / g$. Thus, under the Froude-Krylov approximation, the impulseresponse function corresponding to $H_{f}(i \omega)$ can be expressed as

$$
\begin{aligned}
h_{f}(t) & =\frac{1}{2 \pi} \int_{-\infty}^{\infty} H_{f}(i \omega) \mathrm{e}^{i \omega t} \mathrm{~d} \omega \\
& \approx \frac{1}{2} \rho g R_{s}^{2} \int_{-\infty}^{\infty} \mathrm{e}^{-\omega^{2} / g} \mathrm{e}^{i \omega t} \mathrm{~d} \omega
\end{aligned}
$$

Using Euler's equality,

$h_{f}(t) \approx \frac{1}{2} \rho g R_{s}^{2} \int_{-\infty}^{\infty}\left(\mathrm{e}^{-\omega^{2} / g} \cos \omega t+i \mathrm{e}^{-\omega^{2} / g} \sin \omega t\right) \mathrm{d} \omega$

Since $\sin \omega t$ is an odd function, and $e^{-\omega^{2} / g}$ is an even function of $\omega$, while $\cos \omega t$ is also an even function of $\omega$, the expression above reduces to,

$h_{f}(t) \approx h_{f h}(t)=\rho g R_{s}^{2} \int_{0}^{\infty} \mathrm{e}^{-\omega^{2} / g} \cos \omega t \mathrm{~d} \omega$

defining $h_{f h}$ as the approximate exciting force impulseresponse kernel. The integral in Eq. (51) can be evaluated using integration tables Gradshteyn and Ryzhik (1994), resulting in,

$h_{f h}(t)=\frac{1}{2} \rho g R_{s}^{2} \sqrt{\frac{\pi g}{D_{r}}} \mathrm{e}^{-g t^{2} /\left(4 D_{r}\right)}$

Note that, as expected, $h_{f}(t)$ and its approximation $h_{f h}(\mathrm{t})$ are non-causal functions of $t$. Here, 'exact' variation for the exciting force is defined as that determined using a numerical procedure based on the boundary element method HYDRAN (HYDRAN 2012; Korde and Ertekin 2015). While multipleparameter relationships between the approximate and the exact functions are possible, it is supposed here that, for all $t$, a single multiplicative parameter $\hat{F}$ can be used to relate the approximate and exact functions, where $\hat{F}$ may be updated at every iteration. Thus, in simulations here, the exact $h_{f}(t)$ is thought to be unknown and assumed related to the approximate estimate $h_{f h}(t)$ by

$h_{f}(t)=\hat{F} h_{f h}(t)$

$\hat{F}=F=1$ implies an exact match. It is assumed here that $\hat{F}$ is a slowly varying parameter. 
The radiation impulse-response function $h_{r}$ may generally also be found using a computational procedure, and the odd and even function parts $h_{r}(t)=h_{a}(t)+h_{b}(t)$ would be found simply as $h_{b}(t)=h_{r}(t) / 2, h_{b}(-t)=h_{b}(t)$ for $t>0$, and $h_{a}(t)=h_{r}(t) / 2$, and $h_{a}(-t)=-h_{a}(t)$ for $t>0$. Here, an approximate estimate $h_{r h}$ for $h_{r}$ is obtained based on the Froude-Krylov approximation above i.e incident wave potential much greater than diffraction potential. Using the Haskind-Hanaoka relation Wehausen (1971), the radiation damping in heave can be expressed as,

$b(\omega)=\frac{k\left|F_{f}(i \omega)\right|^{2}}{8 P_{w}} \approx \frac{k}{8 P_{w}}\left|F_{f k}(i \omega)\right|^{2}$

where $P_{w}$ is the incident wave power per unit crest length given by,

$P_{w}=\frac{\rho g^{2} A^{2}(\omega)}{4 \omega}$

Thus, under the Froude-Krylov approximation of Eq. (47),

$b(\omega) \approx b_{h}(\omega)=\frac{1}{2} \rho \pi^{2} k \omega R_{s}^{4} \mathrm{e}^{-2 \omega^{2} D_{r} / g}$

Note that $k=|\omega| \omega / g$, so that $k$ has the same sign as $\omega$. Further, $k$ represents outgoing waves for the radiation problem. Thus, the approximate radiation impulse-response function is defined as,

$h_{r h}(t)=\frac{\rho \pi^{2} R_{s}^{4}}{2 g} \frac{1}{2 \pi} \int_{-\infty}^{\infty}|\omega| \omega^{2} \mathrm{e}^{-2 \omega^{2} D_{r} / g} \mathrm{e}^{i \omega t} \mathrm{~d} \omega$

Using Euler's equality,

$$
\begin{aligned}
h_{r h}(t)= & \frac{\rho \pi^{2} R_{s}^{4}}{2 g} \frac{1}{2 \pi} \int_{-\infty}^{\infty}|\omega| \omega^{2} \mathrm{e}^{-2 \omega^{2} D_{r} / g}(\cos \omega t \\
& +i \sin \omega t) \mathrm{d} \omega
\end{aligned}
$$

Note that, $|\omega| \omega^{2}$ and $\mathrm{e}^{-2 \omega^{2} D_{r} / g}$ are even functions of $\omega$. Since $\cos \omega t$ is an even function and $\sin \omega t$ is an odd function, the integral in Eq. (58) reduces to,

$h_{r h}(t)=\frac{\rho \pi R_{s}^{4}}{2 g} \int_{0}^{\infty}|\omega| \omega^{2} \mathrm{e}^{-2 \omega^{2} D_{r} / g} \cos \omega t \mathrm{~d} \omega$

The integral in Eq. (59) can also be evaluated using integration tables Gradshteyn and Ryzhik (1994). However, it is more convenient to use numerical integration in this case.

Here, the exact $b(\omega)$ is based on a numerical calculation using HYDRAN Korde and Ertekin (2015). Just as with $h_{f h}(t)$ and $h_{f}(t)$, a single (different) multiplicative parameter is assumed to relate the approximate $h_{r h}(t)$ and $h_{r}(t)$.
Thus,

$h_{r}(t)=\hat{R} h_{r h}(t)$

$\hat{R}$ may be updated periodically during the simulation. In simulations, $h_{r h}$ is all that is considered known a priori and the exact $h_{r}$ is thought to be unknown. When $\hat{R}=R=1$, the exact $h_{r}(t)$ is approached. $\hat{R}$ is also assumed to be slowly varying.

\section{References}

Bacelli G, Ringwood JV (2013) A geometric tool for analysis of position and force constraints in wave energy converters. Ocean Eng 65:10 18

Bryson AE, Ho YC (1975) Applied optimal control-optimization, estimation, and control. Hemisphere Publishing Corporation, New York

Budal K, Falnes J (1980) Interacting point absorbers with controlled motion. In: Count B (ed) Power from sea waves. Academic Press, London, pp 381-399

Cretel J, Lightbody G, Thomas G, Lewis A (2011) Maximization of energy capture by a wave-energy point absorber using model predictive control. In: Proc. 18th IFAC World Congress, Milano, Italy, Sept 2011 (preprint)

Cummins W (1962) The impulse response function and ship motions. Schiffstechnik 9:101-109

Evans D (1981) Power from water waves. Ann Rev Fluid Mech 13:157_ 187

Falcaao AFO (2008) Phase control through load control of oscillating body wave energy converters with hydraulic pto system. Ocean Eng 35:358-366

Falcão AFO (2010) Wave energy utilization: a review of the technologies. Renew Sustain Energy Rev 14:899-918

Falnes J (1995) On non-causal impulse response functions related to propagating water waves. Appl Ocean Res 17(6):379-389

Fusco F, Ringwood J (2014) A hierarchical robust control of oscillating wave energy converters with uncertain dynamics. IEEE Trans Sustain Energy 5(3):958-966

Fusco F, Ringwood JV (2010) Short-term wave forecasting for real-time control of wave energy converters. IEEE Trans Sustain Energy 1(2):99-106

Gradshteyn I, Ryzhik I (1994) Table of integrals, series, and products, 5th edn, Alan Jeffrey (ed). Academic Press, San Diego

Hals J, Falnes J, Moan T (2011) A comparison of selected strategies for adaptive control of wave energy converters. J Offshore Mech Arctic Eng 133(3):1-12

Hansen RH, Kramer MM (2011) Modeling and control of the wave star prototype. In: Proc. 9th European Wave and Tidal Energy Conference, Southampton, UK, paper 163

HYDRAN (2012) A computer program for the hydroelastic response analysis of ocean structures. Tech. rep., Offcoast Inc., Kailua, HI, ver. 5.1 .7

Korde U (2015) Near-optimal control of a wave energy device in irregular waves with deterministic-model driven incident wave prediction. Appl Ocean Res 53:31-45

Korde U, Ertekin R (2015) Wave energy conversion by controlled floating and submerged cylindrical buoys. J Ocean Eng Marine Energy 1(3):255-272

Korde U, Robinett R, Wilson D (2015) Approaching maximum power conversion with exergy-based adaptive wave-by-wave control of a 
wave energy converter. In: Proc. MTS/IEEE Oceans Conference, washington, DC

Korde U, Robinett R, Wilson D (2016) Hydrodynamic modeling and control of buoy oscillations for efficient use of wave power in ocean sensing. In: Proc. ONR/MTS Buoy Workshop 2016, Woods Hole, MA

Korde UA (1999) Efficient primary energy conversion in irregular waves. Ocean Eng 26:625-651

Korde UA (2014) On a near-optimal control approach for a wave energy converter in irregular waves. Appl Ocean Res 46:79-93

Mavrakos SA, McIver P (1997) Comparison of methods for calculation of hydrodynamic characteristics of arrays of wave power devices. Appl Ocean Res 19(5,6):283-291

Mei CC (1992) Appl Dyn Ocean Surf Waves. World Scientific, Singapore (Chapter 7)

Naito S, Nakamura S (1985) Wave energy absorption in irregular waves by feedforward control system. In: Evans DV, Falcao AFO (eds) Proceedings of hydrodynamics of wave energy utilization, IUTAM Symp. Springer, Berlin, pp 269-280

NDBC (2016) Station 51207-Kaneohe Bay, HI (198). http://www.ndbc. noaa.gov. June 2016

Robinett R, Wilson D (2007) Collective systems: physical and information exergies. Tech. rep., Sandia National Laboratories, SAND2007-2327
Robinett R, Wilson D (2010a) Nonlinear slewing spacecraft control based on exergy, power flow, and static and dynamic stability. J Astronaut Sci 57(4):717-741

Robinett RD, Wilson DG (2009) Exergy and irreversible entropy production:thermodynamic concepts for nonlinear control design. Int J Exergy 6(3):357-387

Robinett RD, Wilson DG (2010b) Hamiltonian surface shaping with information theory and exergy/entropy control for collective plume tracing. In: International Journal of Systems, Control and Communications 2(1/2/3):144-169, invited paper, special issue on Information Processing and Decision Making in Control Systems

Robinett RD, Wilson DG (2011) Nonlinear power flow control design: utilizing exergy, entropy, static and dynamic stability, and lyapunov analysis. Springer, London

Robinett RD, Dohrmann CR, Eisler GR, Feddema JT, Parker GG, Wilson DG, Stokes D (2002) Flexible robot dynamics and controls. Springer, London

Salter SH (1978) Development of the duck concept. In: Proc. Wave Energy Conference, heathrow, UK

Slotine JJE, Li W (1991) Appl Nonlinear Control. Prentice-Hall, NJ

Wehausen JV (1971) Motion of floating bodies. Ann Rev Fluid Mech 3:237-268

Wehausen JV (1992) Causality and the radiation condition. J Eng Math $26: 153-158$ 